\title{
Modulated surface waves in large-aspect-ratio horizontally vibrated containers
}

\author{
FERNANDO VARAS AND JOSÉ M. VEGA \\ ËIS lngenieros de 'lelecomunicación, Universidad de Vigo, Campus Marcosende, \\ 36280 -Vigo, P'ontevedra, Spain \\ ГTS Ingenieros Aeronáuticos, Universidad Politécnica de Madrid, Plaza Cardenal \\ Cisneros, 3, 28040-Madrid, Spain
}

We consider the harmonic and subharmonic modulated surface waves that appear upon horizontal vibration along the surface of the liquid in a two-dimensional large-aspect-ratio (length large compared to depth) container, whose depth is large compared to the wavelength of the surface waves. The analysis requires us also to consider an oscillatory bulk flow and a viscous mean flow. $\Lambda$ weakly nonlincar description of the harmonic waves is made which provides the threshold forcing amplitude to trigger harmonic instabilities, which are of various qualitatively different kinds. A linear analysis provides the threshold amplitude for the appearance of subharmonic waves through a subharmonic instability. The results obtained are used to make several specific qualitative and quantitative predictions.

\section{Introduction}

Surface waves in vibrated containers have received much attention in the literature, largely focused on Faraday waves (Faraday 1831; Rayleigh 1883; Miles \& Henderson 1990; Cross \& Hohenberg 1993; Fauve 1995), which are excited upon vertical vibrations. IIorizontally excited waves have received less attention and workers have concentrated on the casc of containers with a moderate width (Milcs 1984; Funakoshi \& Inoue 1988; Nobili el al. 1988; Feng 1997; Billingham 2002; IIill 2003; Faltinsen, Rognebakke \& Timokha 2006), whose horizontal size is comparable to the surface waves wavelength. In this casc, only a few (depending on symmetrics) sloshing modes are excited; note that depth plays no role in this discussion, and only affects the eigenmodes quantitatively. If mean flows are ignored, then the system is governed by a set of ODIs. Mean flows are due to time-averaged quadratic nonlinearities that produce both a global circulation and a deformation on the free-surface elevation. The former in turn can be associated cither to viscous effects (viscous mean flows) or to mass conservation (inviscid mean flows) and has been largely ignored in the analysis of surface waves in moderate containers. Surface waves have been analysed usually from a IIamiltonian formulation, with viscous effects added a posteriori through a linear viscous damping, ignoring some more subtle, but equally important, nonlinear viscous effects. Viscous mcan flows (also called strcaming llows, Schlichting 1968; Rilcy 2001), in particular, are produced by time-averaged Reynolds stresses in the oscillatory boundary layers, and have been mistakenly assumed to be only a byproduct of surface waves; instead, viscous mean flows have been proved to couple dynamically with the evolution of the primary surface waves (see Higuera, Vega \& Knobloch 2002 and 
references therein). The mean free-surface deformation instead has been considered in connection with the so-called vibroequilibria (Gavrilyuk, Lukovsky \& Timokha 2004 and references therein) and also in connection with dynamic stabilization of, for example, Rayleigh-Taylor instability (Wolf 1969; Lapuerta, Mancebo \& Vega 2001 and references therein).

For wider containers (namely, containers whose width is large compared to wavelength), we must consider a large number of surface modes and interaction with noarby modes, which leads to spatial modulation. 'This can be analysed invoking separation of scales using a description of the modulating amplitude in terms of (Schrödinger-like cquations with convective terms resulting from group velocity) PDГs. Spatially modulated surface waves have received much less attention (two exceptions, with mean flows ignored are Ockendon \& Ockendon 1973; Faltinsen \& l'imokha 2002) even though some related experiments (Wuncnburger et al. 1999) suggest that they exhibit rich dynamics. Let us remark that Wunenburger et al. deals with a $\mathrm{CO}_{2}$-system near the critical point. It is somewhat surprising that the simpler ordinary liquid problem has been analysed neither theoretically nor experimentally; efforts have concentrated in both two-phase (Kozlov 1991, Ivanova, Kozlov \& Evesque 1996) and granular flows (Jacger \& Nagel 1996; Ristow 2000; Aranson \& 'l'simring 2006). Spatially modulated surface waves are also interesting from the theorical point of view because the flow field has a fairly rich structure. As is to be expected, vibrations produce surface waves near the free surface, but also an oscillatory flow in the bulk and a mean flow. The analysis of these is non-trivial for several reasons.

(i) Surface waves are spatially modulated, countcrpropagating waves that are described by two coupled Shrödinger-like amplitude equations. In addition to the usual effects of dispersion and conservative nonlinearity, these equations exhibit new terms accounting for (a) viscous dissipation, $(b)$ forcing, (c) advection at the group velocity, and $(d)$ coupling to the mean flow. The advection term is always much larger than the dispersive term, and (because both counterpropagating waves are present) cannot be eliminated by using a moving frame, as is done when only one-sided waves are considered, for example, in water wave theory (Newell 1985). If dispersion is neglected, then the equations become hyperbolic, as in related dissipative systems (first considered by Danicls 1978, sce also Martcl \& Vega 1998), where small diffusive terms lead to subtle effects (Martel \& Vega 1996). Similarly, dispersion cannot be ignored a priori in counterpropagating surface waves (Lapuerta, Martel \& Vega 2002; Martel, Vega \& Knobloch 2003) because dispersive scales can be destabilized. 'l'here are two kinds of surface waves, namely harmonic and subharmonic, whose frequency is the forcing frequency and hall of the forcing frequency, respectively.

(ii) The horizontally vibrating lateral walls act as wavemakers, and directly excite a pair of harmonic surface waves (HSW), analysed in $\$ 4$, which travel along the free surface from the lateral walls inwards and decay from surface dissipation. Forcing at the lateral walls is not standard. This is because the walls extend down to the bottom of the container (which makes a difference with standard wavemakers in water waves) and thus they also excite an internal oscillatory flow in the bulk (OBF, analysed in $\$ 3$ ), which affects a region whose size is of the order of the container's depth and contributes to the excitation of the surface waves in a subtle manner. Namely, it produces:

(a) A contribution (namely, that term proportional to $\ln d$ in (4.16) below) to direct excitation of harmonic waves at the lateral walls that is logarithmically large if the container depth is large compared to wavelength. This contribution has not been considered before in this context. 


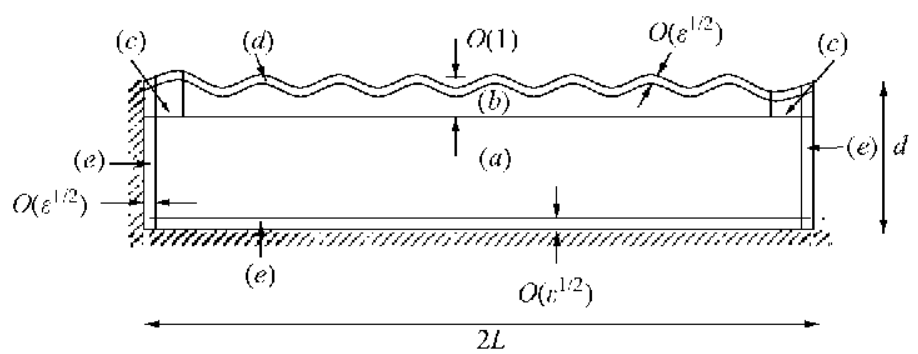

FIGURL: 1 . Sketch of the fluid domain, with the various asymptotic regions. The contact line is pinned to the upper edge of the lateral walls.

(b) $\Lambda \mathrm{n}$ oscillatory pressure gradient on the free surface that is able to excite parametrically (Tauve 1995) new subharmonic surface waves (SSW), analysed in $\$ 5$.

(iii) Surface waves excite a secondary viscous mean flow (VMF), which affects the weakly nonlinear dynamics of the primary waves, as repeatedly shown in related vibrating fluid systems (Higuera et al. 2002; Lapucrta et al. 2002, and references therein), and is at least as strong as the inviscid mean flow usually considered in large-aspect-ratio surface waves (Davey \& Stewartson 1974). The effect of the VMF on the harmonic surface waves will be considered in $\$ 4$, where it will be secn that this effect cannot be ignored when considering the stability of the primary waves. Similarly, the VMF aflects the weakly nonlincar dynamics of subharmonic surface waves, but the analysis of this is beyond the scope of this paper.

Against this background, the object of this paper is to analyse the dynamics of the spatially modulated ncarly inviscid surface waves in a long container subject to horizontal vibration. In order to avoid additional difficulties and to clarify the analysis, we consider the restricted case of one-dimensional waves in a two-dimensional container, which models waves in a three-dimensional rectangular container whose width is small compared to length, but large compared to depth. $\Lambda$ lso, we assume a fixed contact line to avoid additional effects due to contact line hysteresis; see Henderson \& Miles 1994; Bechhoefer et al. 1995 for the experimental realization of this condition.

\section{Formulation}

We consider a two-dimensional horizontal container (figure 1) of depth $d^{*}$ and length $2 L^{*}$, which is vibrating horizontally with an amplitude $a^{*}$ and a frequency $\omega^{*}$. We use for non-dimensionalization the characteristic time $\omega^{*-1}$ and characteristic length $\ell^{*}$, defined by the inviscid capillary gravity waves dispersion relation in deep layer

$$
\omega^{* 2}=s / \ell^{*}+\sigma /\left(\rho \ell^{* 3}\right)
$$

in terms of the acceleration due to gravity $g$, surface tension $\sigma$, and density $\rho$, all assumed constant. The resulting non-dimensional governing equations are

$$
\psi_{x x}+\psi_{y y}=\Omega, \quad \Omega_{t}-\psi_{y} \Omega_{x}+\psi_{x} \Omega_{y}=\varepsilon\left(\Omega_{x x}+\Omega_{y y}\right),
$$

in $-L<x<L,-d<y<f$. The boundary conditions at the free surface are

$$
f_{i}-\psi_{x}-\psi_{y} f_{x}=0, \quad\left(\psi_{y y}-\psi_{x x}\right)\left(1-f_{x}^{2}\right)-4 f_{x} \psi_{x y}=0,
$$




$$
\begin{aligned}
(1 & -S) f_{x}-S\left(\frac{f_{x}}{\sqrt{1+f_{x}^{2}}}\right)_{x x}-\psi_{y z}+\psi_{x t} f_{x}-\left(\psi_{x}+\psi_{y} f_{x}\right) \Omega+\frac{1}{2}\left(\psi_{x}^{2}+\psi_{y}^{2}\right)_{x} \\
& +\frac{1}{2}\left(\psi_{x}^{2}+\psi_{y}^{2}\right)_{y} f_{x}=-\varepsilon\left[3 \psi_{x x y}+\psi_{y y y}-\left(\psi_{x x x}+\psi_{x y y}\right) f_{x}\right] \\
& +2 \varepsilon\left[\frac{2 \psi_{x y} f_{x}^{2}+\left(\psi_{x x}-\psi f_{y y}\right) f_{x}}{1+f_{x}^{2}}\right]_{x}+2 \varepsilon \frac{\left(\psi_{x x y}-\psi_{y y y}\right) f_{x}^{2}-\psi_{x y y}\left(1-f_{x}^{2}\right) f_{x}}{1+f_{x}^{2}}
\end{aligned}
$$

at $y=f$, while those at the bottom and lateral walls are

$$
\begin{gathered}
\psi r=0, \quad \psi_{y}=a \cos t \quad \text { at } y=-d \\
\psi_{x}=0, \quad \psi_{y}=a \cos t, \quad f=0 \quad \text { at } x= \pm L-a \sin t
\end{gathered}
$$

Here, $\psi$ is the streamfunction, defined such that the velocity

$$
(u, v)=\left(-\psi \psi_{y}, \psi_{x}\right) \text {; }
$$

$\Omega=v_{y}-u_{x}$ is the vorticity, and $f$ is the frec-surface clevation, required to satisfy volume conservation $\int_{L}^{L} f \mathrm{~d} x=0$. Equations (2.2)-(2.6) arc oblained in a standard way, replacing (2.8) and $\Omega$ into the usual continuity and Navier-Stokes equations, and the usual boundary conditions (accounting for kincmatic compatibility and equilibrium of tangential and normal stresses at the free surface, and no slip at the bottom).

'The problem depends on the following non-dimensional parameters: the container's length $2 L$ and depth $d$, the forcing amplitude $a$, the capillary-gravity balance parameter $S$, and the non-dimensional viscosity $\varepsilon$, defined as

$$
(L, d, a)=\frac{\left(L^{*}, d^{*}, a^{*}\right)}{\ell^{*}}, \quad S=\frac{\sigma}{\sigma+\rho g \ell^{* 2}}, \quad \varepsilon=\frac{v}{\omega^{*} \ell^{* 2}},
$$

where $\nu$ is the kinematic viscosity. Note that $S=1 /(1+B)$, where $B=\rho g \ell^{* 2} / \sigma$ is the Bond number based on the wavelength of the surface waves, $\ell^{*}$, defined in (2.1). $S$ is such that $0 \leqslant S \leqslant 1$, and the extreme values $S=0$ and 1 correspond to the purely gravitational $(\sigma=0)$ and the purcly capillary $(g=0)$ limits, respectively.

The problem (2.2)-(2.6) is invariant under the action

$$
x \rightarrow-x, \quad \psi r \rightarrow-\psi, \quad \Omega \rightarrow-\Omega, \quad t \rightarrow t+\pi,
$$

which results from reflection symmetry, and will play an important role below.

I'he assumptions in this paper are

$$
\varepsilon \ll 1, \quad 1 \ll d \ll L, \quad a \ll 1,
$$

which means that (i) viscous effects are weak, (ii) the horizontal length is large compared to depth, which in turn is large compared to the wavelength of the surface waves. T'he assumptions that $: \ll 1, a \ll 1$ and $L \gg 1$ are cssential in the analysis below, but the remaining assumptions are made only to simplify presentation, and could be relaxed. In fact, the assumption that $L \gg 1$ is only required in $\S 4$, where it is made in order that the viscous mean flow be almost parallel. The analysis in $\$ \$ 35$ will be made in specific distinguished limits, which involve relations between the small parameters and will be defined such that as many terms as possible in the resulting asymptotic equations be of the same order. I Iowever, the analysis will be valid for arbitrary values of the small parameters satisfying $(2.10)$; the only additional restrictions (validity limits of the analysis) will be that the coefficients of the asymptotic equations be bounded, which will exclude some resonances, see below. 
In principle, we should consider several regions in the container (see figure 1). (a) The bulk, which is that part of the container outside the several boundary layers described next, $(b)$ a ncarly inviscid frec surface boundary layer, with a $O(1)$ thickness, which is the region affected by the surface waves, $(c)$ two nearly inviscid corner regions near the contact point, with $O(1)$ size, where the surface waves are directly forced and reflected, $(d)$ a viscous free-surface boundary layer, with an $O\left(\varepsilon^{1 / 2}\right)$ thickness, and $(e)$ a viscous boundary layer near the lateral walls and the bottom of the container, whose thickness is also $O\left(\varepsilon^{1 / 2}\right)$. Becausc $d$ and $L$ are both large, region $(e)$ has a negligible effect in the analysis below. The assumption that $d \ll L$ will only be required in $\$ 4$ and relaxed in the remainder of the paper. All calculations below will be nearly inviscid. Viscous effects come into play only through the VMГ mentioned above and the damping ratio of the various wavetrains. In order to calculate the latter, we recall the well-known result (Miles \& Henderson 1990) that a resonant surface wave whose free-surface elevation is given by $f=A \mathrm{e}^{\mathrm{i}(\omega t \pm k x)}+$ c.c., where $\omega$ and $k$ satisfy the dispersion relation (cf. (2.1))

$$
\omega=\omega(k) \equiv \sqrt{(1-S) k+S k^{3}},
$$

exhibits a damping ratio

$$
2 k^{2} \varepsilon
$$

As explained in $\$ 1$, we must consider an oscillatory bulk flow (OBF), the harmonic and subharmonic surface waves (HSW and SSW), and a viscous mean flow (VMF). l'hus

$$
\psi=\psi_{O B F}+\psi_{H S W}+\psi_{S S W}+\psi_{Y M F}, \quad f=f_{O B F}+f_{H S W}+f_{S S W}+f_{M M F},
$$

where $\left(\psi_{O B H}, \int_{O B H}\right),\left(\psi_{I I S W}, \int_{I I S W}\right)$, and $\left(\psi_{S S W}, \int_{S S W}\right)$ are given by $(3.1),(3.6),(4.1)-(4.3)$ and (5.1) below. To the approximation relevant in this paper, the VMF can be ignored except in the analysis of the HSW.

Note that the problem exhibits four well-separated spatial scales, or orders $O\left(\varepsilon^{1 / 2}\right)$, $O(1), O(d)$ and $O(L)$, and the associated timc scalcs. Since, in addition, this is a free-boundary problem, direct numerical simulation is difficult. The analysis below instead filters out all spatial and temporal scales except for the largest ones.

The remainder of the paper is organized as follows. The OBF is calculated in $\$ 3$, as we need to obtain its effect in promoting harmonic and subharmonic waves. The HSW are analysed in $\$ 4$, where a system of weakly nonlinear amplitude equations coupled to the VMF is derived which is used in $\$ 4.1$ to calculate the simplest reflection symmetric modulated waves and in $\$ 4.2$ to analyse the linear stability of these. The latter analysis provides the threshold amplitude for the appearance of harmonic instabilities of the system. The $\mathrm{SSW}$ are considered in $\$ 5$, where a linear stability analysis is made that provides the amplitude threshold for the parametric excitation of these waves, through a subharmonic instability. This is made in three distinguished regimes, depending on the relation between the container's depth and length and the viscous length; a more general, weakly nonlinear analysis of this flow is outside the scope of this paper. IIarmonic and subharmonic instabilities are compared in $\$ 6$, to elucidate which instability dominates for each set of parameter values; some specific predictions are made and some experiments are suggested. Finally, the results are summarized and discussed in $\$ 7$. 


\section{Oscillatory bulk flow}

The oscillatory bulk flow is produced by the lateral walls oscillations, and has not becn considered before in this context. I his flow affects both reflection at the lateral walls (considered in Appendix A) and excitation of subharmonic waves (considered in $\$ 5$ ). To the approximation relevant in this paper, only the leading-order approximation (strictly inviscid and linear) in region $(a)$ is necessary; note that the solution in the smaller regions $(b)(e)$ is slaved to the solution in region $(a)$, which is always the case in nearly inviscid oscillatory flows when only leading-order terms are considered. 'the streamfunction in this region is given by

$$
\psi_{O B F}=a d \varphi(\zeta, \eta) \cos \iota,
$$

where the rescaled spatial variables and the aspect ratio are defined as

$$
(\zeta, \eta)=\frac{(x, y)}{d}, \quad \Lambda=\frac{L}{d} .
$$

Substituting thesc into $(2.2 a),(2.4),(2.5 a),(2.6 b, c)$, and sctling $\Omega=\varepsilon=0$, we obtain

$$
\begin{gathered}
\varphi_{\zeta \zeta}+\varphi_{\eta \eta}=0 \quad \text { in }-\Lambda<\zeta<\Lambda, \quad-1<\eta<0, \\
\varphi_{\eta}=0 \quad \text { at } \eta=0, \quad \varphi=0 \quad \text { at } \eta=-1, \quad \varphi_{\eta}=1 \quad \text { at } \zeta= \pm \Lambda,
\end{gathered}
$$

where the kincmatic boundary condition (2.3a) has not been used. The boundary condition at $\eta=0$ results from (2.4) (anticipating that $f \sim a$, see (3.6) below) and imposes a valuc of zero on the horizontal velocity there. 'this is because no term in (2.4) can balance $\psi_{y t}$ (accounting for pressure perturbations associated with free-surface deformation), which makes a difference between the oscillatory bulk flow considered here (which exhibits an $O(d)$ characteristic length) and surface waves, where pressure perturbations are balanced by capillary gravity effects. The boundary conditions $(2.3 b)$ and $(2.5 b)$ cannot be imposed in this inviscid approximation, but could be accounted for considering two oscillatory boundary layers.

The (unique) solution of (3.3)-(3.4) is such that $\varphi$ is an even function of $\zeta$. Equations (3.3)-(3.4) are readily solved upon separation of variables, as

$$
\varphi=\sum_{n=0}^{\infty} a_{n} \frac{\cosh \left(\lambda_{n} \zeta\right)}{\cosh \left(\lambda_{n} \Lambda\right)} \sin \left[\lambda_{n}(\eta+1)\right]
$$

where $\lambda_{n}=(2 n+1) \pi / 2$ and $a_{n}=2(-1)^{n} / \lambda_{n}^{2}$ are such that $\sum a_{n} \sin \left[\left(\lambda_{n}(\eta+1)\right]=\eta+1\right.$ in $-1<\eta<0$. The free-surface elevation follows using $(2.3 a)$,

$$
f_{O B F}=a \varphi_{\zeta}(\zeta, 0) \sin t
$$

and exhibits a logarithmic singularity at $\zeta= \pm \Lambda$. This is seen noting that the vertical velocity at the free surface (which in turn is proportional to the vertical pressure gradient)

$$
\varphi_{\zeta}(\zeta, 0) \equiv \sum_{n=0}^{\infty} \frac{2}{\lambda_{n}} \frac{\sinh \left(\hat{\lambda}_{n} \zeta\right)}{\cosh \left(\lambda_{n} \Lambda\right)} \simeq \mp\left[\frac{2}{\pi} \ln (\Lambda \mp \zeta)-H(\Lambda)\right] \quad \text { as } \zeta \rightarrow \pm \Lambda .
$$

where the function $I I$ (plotted in figure 2) is given by

$$
H(\Lambda)=\frac{2}{\pi} \ln \frac{4}{\pi}-2 \sum_{n=0}^{\infty} \frac{\exp \left(-\lambda_{n} \Lambda\right)}{\lambda_{n} \cosh \left(\hat{\lambda}_{n} \Lambda\right)}
$$




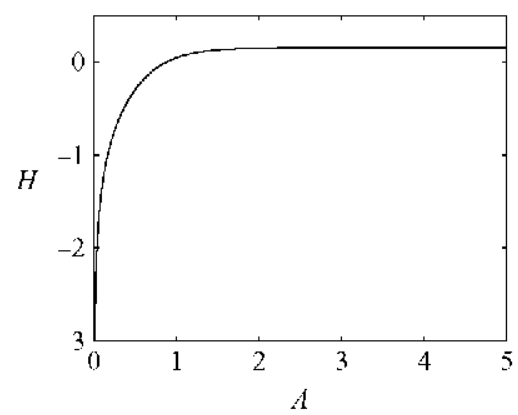

FIGURL 2. The function $I(\Lambda)$ defined in (3.8).

and is such that

$$
H(\Lambda) \rightarrow H(\infty)=\frac{2}{\pi} \ln \frac{4}{\pi} \simeq 0.154 \text { as } \Lambda \rightarrow \infty .
$$

Because of this singularity, the solution above breaks down as $\zeta \rightarrow \pm \Lambda$, which leads us to region (c), analysed in $A$ ppendix $A$.

Note that the analysis above does not require that the aspect ratio $A$ be large. As $\Lambda \rightarrow \infty, \varphi$ vanishes exponentially except in two lateral regions, where $(| \pm \zeta-\Lambda| \sim 1$ and $\varphi=\sum a_{n} \exp \left[\left(\hat{\lambda}_{n}( \pm \zeta-\Lambda)\right] \sin \left[\lambda_{n}(\eta+1)\right]\right.$.

\section{Harmonic surface waves at large aspect ratio: $L \gg d$}

These are a pair of counterpropagating waves that are forced (and reflected) in region $(c)$ and propagate along region (b) (figure 1). The description below is weakly nonlinear, and must include the effect of the viscous mean flow, whose analysis is greatly simplificd at large aspect ratio. 'l'hus, the assumption that $L \gg d$ will be used below. We shall consider the limit (2.10), with $d$ only logarithmically large, such that $\mathrm{e}^{2 d} \ll 1$. In this case, $d$ can be treated as $O(1)$.

$\Lambda$ ccording to the non-dimensionalization defined in $\$ 2$, the non-dimensional forcing frequency is 1 . Then, the inviscid dispersion relation (2.11) shows that the nondimensional wavenumber is also (close to) 1 . In fact, in order to include the effect of the spatial detuning ( $\delta$, given by $(4.16)$ below) resulting from the phase shift produced by reflection at the lateral walls, we shall correct this value of $k$ by an amount $\delta / L \ll 1$. 'lhus, the frec-surface clcvation and the streamfunction associated with the harmonic surface waves in region $(b)$ are written as

$$
\begin{aligned}
& f_{\text {IISW }}=\sqrt{\varepsilon} \mathrm{e}^{\mathrm{i} i}\left[A^{-} \mathrm{e}^{\mathrm{i}(1+\delta / I .) x}+A^{-} \mathrm{e}^{-\mathrm{i}(1-\delta / L / \mathrm{x}}\right]+\text { c.c. }+\cdots,
\end{aligned}
$$

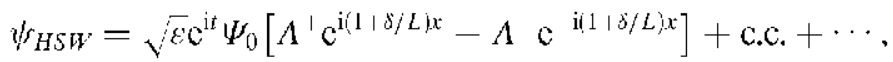

where $\Psi_{0}=\mathrm{c}^{y}$. These waves are coupled to the viscous mean flow, whose associated free-surface elevation and streamfunction are

$$
f_{V M H}=\varepsilon f^{m}, \quad \psi_{Y M H}=\varepsilon \psi^{m} .
$$

Ihe complex amplitudes $\Lambda^{\perp}$ have becn rescaled (with $\sqrt{\varepsilon}$ ) imposing that the viscous damping term and the cubic nonlinearity be comparable in the amplitude equations (4.5) below; $f^{m}$ has been rescaled (with $\varepsilon$ ) anticipating that the mean flow is quadratic in the complex amplitudes. Both $A-$ and $f^{m}$ depend only on the following slow space and time variables, defined such that the time derivative and that term 
accounting for advection at the group velocity be both comparable to the viscous damping term in the amplitude equations (4.5) below,

$$
\xi=\varepsilon x, \quad \tau=\varepsilon \ell,
$$

while $y^{m}$ is also allowed to depend on $y$.

The complex amplitudes $A^{ \pm}$, and the mean flow variables, $\psi^{n}$ and $f^{m}$, evolve according to the following system of coupled amplitude-mean flow equations

$$
\begin{aligned}
A_{\tau}^{\perp} \mp v_{g} A_{\xi}^{ \pm}= & \mathrm{i} \varepsilon \alpha_{0} A_{\xi \xi}^{ \pm}+\left(\mathrm{i} v_{g} \delta / \hat{L}-2\right) A^{-} \\
& +\mathrm{i}\left(\alpha_{1}\left|A^{ \pm}\right|^{2}-\alpha_{2}\left|A^{\mp}\right|^{2}\right) A^{ \pm} \pm 2 \mathrm{i} \int_{-d}^{0} \mathrm{e}^{2 y} \psi_{y}^{n !} \mathrm{d} y A^{ \pm}, \\
\psi_{y y \tau}^{n}= & \psi_{y y y}^{m} \quad \text { in }-d<y<0,
\end{aligned}
$$

with boundary conditions

$$
\begin{gathered}
\psi_{\xi}^{m}-f_{\tau}^{n}=2\left(\left|A^{-}\right|^{2}-\left|A^{-}\right|^{2}\right)_{\xi}, \quad \psi_{y y}^{m}=8\left(\left|A^{+}\right|^{2}-\left|A^{-}\right|^{2}\right) \quad \text { at } y=0, \\
(1-S) f_{\xi}^{m}-\psi_{y \tau}^{m}+\psi_{y y y}^{m}=0 \quad \text { at } y=0, \\
\psi^{m}=\psi_{y}^{m}=0 \quad \text { at } y=-d
\end{gathered}
$$

These cquations and boundary conditions are obtaincd substituting (3.1)-(3.2), (3.5)(3.6), (4.1)-(4.4) and $\left(\psi_{S S W}, f_{S S W}\right)=(0,0)$ into (2.13), and the resulting expressions into (2.2) (2.4), and applying solvability conditions associated with those resonant terms that either depend on the short time variable as $\mathrm{e}^{\mathrm{i} \perp \perp i x}$ (harmonic surface waves) or are independent of the short time variable (mean flow). This is involved and requires us also to consider the solution in regions $(a)$ and $(d)$, as done for modulated Faraday waves (which also involve parametric forcing) by Lapuerta et al. (2002); see also Vega, Knobloch \& Martel (2001) for a more detailed derivation of (4.5) (4.9) in a more general setting. It follows that the group velocity and the coefficients $\alpha_{0}, \alpha_{1}$ and $\alpha_{2}$ are

$$
\begin{array}{r}
v_{g}=\omega^{\prime}(1) \equiv(1+2 S) / 2, \quad \alpha_{0} \equiv-\frac{\omega^{\prime \prime}(1)}{2}=\frac{(1+2 S)^{2}}{8}-\frac{3 S}{2}, \\
\alpha_{1}=\frac{3 S}{1-3 S}+\frac{8-3 S}{4}, \quad \alpha_{2}=\frac{2}{1+3 S}+\frac{4+3 S}{2} .
\end{array}
$$

These are plotted vs. $S$ in figure 3. Note that $\alpha_{1}$ diverges at $S=1 / 3$, where a secondorder internal resonance takes place, and the coefficient of $f_{\xi}^{m}$ vanishes at $S=1$, where capillarity can no longer be ignored. We avoid these resonances assuming that

$$
S \neq \frac{1}{3}, \quad S \neq 1 .
$$

The boundary conditions at the lateral walls are

$$
\begin{gathered}
A^{\perp}=A^{1}+\Phi_{1} \hat{a} \quad \text { at } \xi= \pm \hat{L}, \\
\Lambda_{\xi}^{\perp}+\Lambda_{\xi}=\mp\left(\frac{\mathrm{i} \delta}{\hat{L}}-\frac{2}{v_{g}}\right) \Phi_{1} \hat{a}-\frac{2 \mathrm{i}}{v_{g}} \int_{a}^{0} \mathrm{c}^{2 y} \Psi_{y}^{m} \mathrm{~d} y\left(\Lambda^{1}+\Lambda\right) \\
-\frac{\mathrm{i}}{v_{g}}\left|A^{+}\right|^{2}\left(\alpha_{1} A^{+}+\alpha_{2} A^{-}\right)+\frac{\mathrm{i}}{v_{g}}\left|A^{-}\right|^{2}\left(\alpha_{2} A^{+}+\alpha_{1} A^{-}\right) \quad \text { at } \xi= \pm \hat{L}, \quad \text { (4.13) } \\
\psi^{m}=2\left(|\Lambda|^{2}-\left|\Lambda^{1}\right|^{2}\right) \quad \text { at } y=0, \xi= \pm \hat{L}, \quad \int_{\hat{L}}^{\hat{L}} f^{m}(\xi, \tau) \mathrm{d} \xi=0, \quad(4.14 a, b)
\end{gathered}
$$




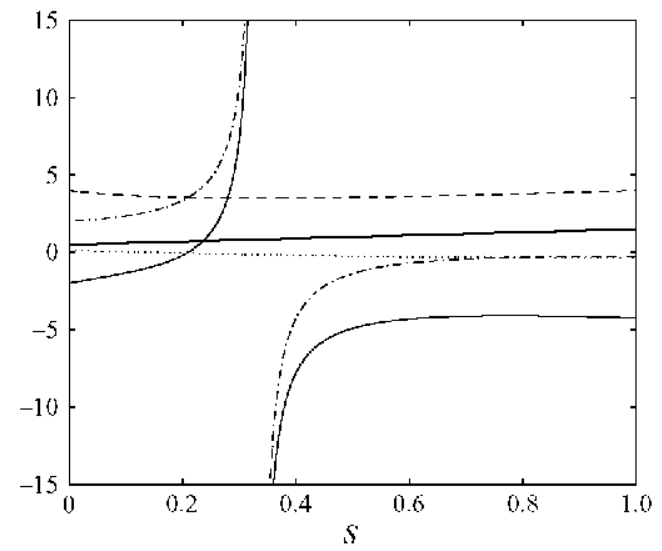

FIGi:RF 3. The quantities $v_{g}(-), \alpha_{0}(\cdots), \alpha_{1}(\cdot), \alpha_{2}(\quad)$, delined in (4.10), and $\alpha_{1}-\alpha_{2}$ ) in terms of $s$.

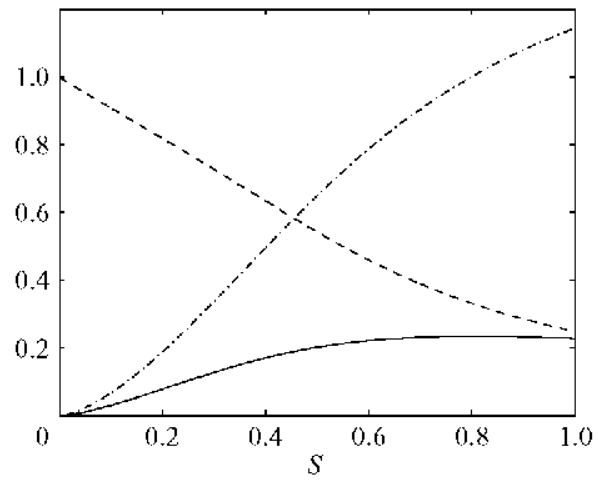

FIGURE 4. The quantities (a) $G_{1}\left(-, G_{2}(--)\right.$ and $\alpha(-\cdot-)$ appearing in (4.16).

where the re-scaled forcing amplitude $\hat{a}$ and container length $\hat{L}$ are defined as

$$
\hat{a}=\frac{a}{\varepsilon^{1 / 2}}, \quad \hat{L}=\varepsilon L .
$$

The spatial detuning $\delta$ and the cocficient $\Phi_{1}$ are

$$
\delta=L-\alpha(S)(\bmod 2 \pi), \quad \Phi_{1}(S, d)=G_{1}(S)\left[\frac{\ln d}{\pi}-\frac{H(\infty)}{2}\right]+G_{2}(S),
$$

where the quantity $H(\infty)$ is given in (3.9) and the quantities $\alpha, G_{1}$ and $G_{2}$ are given in $(\Lambda 11)$ in $\Lambda$ ppendix $\Lambda$ and are plotted $v s . S$ in figure 4.

The boundary condition (4.12) at $\xi=\hat{L}$. (that at $\xi=-\hat{L}$. follows by reflection symmetry) results from matching conditions with the solution in region (c), calculated in Appendix A. This boundary condition accounts for wave reflection (the term $A^{\perp}$ on the right-hand side) and forcing at the lateral walls (the term $\Phi_{1} \hat{a}$ ). The boundary condition (4.13) must be imposed because since we are including dispersion, (4.5) is second order in $\xi$. As explained by Martel et al. (2003) (see also Martel \& Vega 1996), the appropriate additional boundary condition is the compatibility condition for the hyperbolic equations obtained when dispersion is ignored, which in our case is precisely (4.13). Equation (4.14a) at $\xi= \pm \hat{L}$ imposes no net mass flux across the 
lateral sidewalls. To explain this, we must take into account that mass is transported by the mean flow with the so-called mass-transport (or Lagrangian) velocity, whose horizontal component, $u^{m i}$, is obtained by adding the Stokes drift to the Eulcrian velocity considered above, $-\psi_{y}^{m}$. I Jsing standard formulae (Batchelor 1967), we obtain

$$
u^{m t}=-v_{y}^{m}-4\left(\left|\Lambda^{1}\right|^{2}-|\Lambda|^{2}\right) \mathrm{c}^{2 y} .
$$

Equation (4.14a) is obtaincd by simply imposing $\int_{d}^{0} u^{m t} \mathrm{~d} y=0$ at $\xi= \pm \hat{L}$ (and neglecting $\mathrm{e}^{d}$, which is small). Equation (4.14b) results from volume conservation.

The remainder of this section is devoted to the analysis of (4.5)-(4.9) and (4.12)(4.14). Ilowever, some remarks on these are now in order.

(i) We are including a higher-order term in (4.5), namely that term proportional to $A_{\zeta \zeta}$, which is due to dispersion and is responsible for short-wave instabilities, see below.

(ii) There is an additional requirement for the validity of the expansion above, namely that $v_{z} \neq \sqrt{(1-S) d}$ (= the phase velocity of some slowly varying internal waves associated with the mean flow, see Appendix C) or, using (4.10), that

$$
S \neq \frac{\sqrt{d^{2}+6 d}-1-d}{2} .
$$

(iii) Equations (4.5) (4.9) and (4.12) (4.14) are invariant under the following action, which results from the reflection symmetry (2.9),

$$
\xi \rightarrow-\xi, \quad A^{-} \leftrightarrow A^{-}, \quad \psi^{m} \rightarrow-\psi^{m} .
$$

(iv) The counterpart of the amplitude equation (4.5) for the liquid bridge geometry in the lincar approximation $\left|\Lambda^{ \pm}\right| \sim \hat{a} \ll 1$ (the mean flow can be neglected in this limit) was considered by Nicolás, Rivas \& Vega (1998), with quite good comparison with exact solutions.

\subsection{Reflection-symmetric steady states}

The reflection-symmetric (invariant under (4.19)) steady states of (4.5) (4.9) and (4.12)-(4.14) build the primary branch of solutions, and can be written as

$$
A^{ \pm}=R^{ \pm} \mathrm{e}^{\mathrm{i} \ell \cdot} \quad \text { with } \quad R^{-}(\xi)=R^{+}(-\xi), \quad O^{-}(\xi)=0^{+}(-\xi) .
$$

Non-symmetric steady states can be calculated in a similar manner, but they appear only in bifurcations from the primary branch, see below.

Integration of (4.6)-(4.14) yields

$$
\begin{aligned}
\psi^{m} & =\frac{\left(2 d^{2}+1\right) y-2 d}{d^{3}}(y+d)^{2}\left\lfloor\left(R^{+}\right)^{2}-\left(R^{-}\right)^{2}\right], \\
f_{\xi}^{m} & =\frac{6\left(2 d^{2}+1\right)\left[(R)^{2}-\left(R^{\prime}\right)^{2}\right]}{d^{3}(1-S)} .
\end{aligned}
$$

Note that the surface waves produce a non-zcro steady-surface deflection (which could be obtained integrating (4.22) and imposing that $\int^{m}=0$ at $\xi= \pm \hat{L}$ ). This is related to the so-called vibroequilibria (Gavrilyuk et al. 2004), but in a more general setting (the viscous mean flow is usually ignored in vibroequilibria analyses, but affects the quantitative value of $f^{m}$ here). Substituting (4.20) (4.22) into (4.5), and neglecting 
both $O(\varepsilon)$-terms and $O\left(\mathrm{e}^{-2 d}\right)$-terms, we obtain

$$
\begin{aligned}
& \mp v_{g} R_{\xi}^{\perp}=-2 R^{ \pm}, \\
& \mp v_{g} \theta_{\xi}^{ \pm}=\frac{v_{g} \delta}{\hat{L}}+\alpha_{1}\left(R^{\perp}\right)^{2}-\alpha_{2}\left(R^{1}\right)^{2} \pm \beta_{0}\left\lfloor\left(R^{+}\right)^{2}-\left(R^{-}\right)^{2}\right\rfloor, \\
& R^{ \pm} \mathrm{e}^{\mathrm{i} \theta^{\prime}}=R^{\mp} \mathrm{e}^{\mathrm{i} \theta^{\mp}}+\Phi_{1} \hat{a} \quad \text { at } \xi= \pm \hat{L},
\end{aligned}
$$

where

$$
\beta_{0}=\frac{4 d^{4}-8 d^{3}+3}{2 d^{3}}
$$

Integration of these cquations invoking $(4.20 b, c)$ yiclds

$$
\begin{aligned}
& R^{-}=R_{0} \exp \left( \pm 2(\xi \mp \hat{L}) / v_{g}\right), \\
& \theta^{-}=\theta_{0} \mp \frac{\delta \xi}{\hat{L}}-\frac{\left(\alpha_{1}+\beta_{0}\right) R_{0}^{2} \exp \left( \pm 4(\xi \mp \hat{L}) / v_{g}\right)+\left(\alpha_{2}+\beta_{0}\right) R_{0}^{2} \exp \left(\mp 4(\xi \pm \hat{L}) / v_{g}\right)}{4},
\end{aligned}
$$

where $R_{0}$ and $\theta_{0}$ are given by the following equations, which are obtained upon substitution of (4.22) (4.25) into (4.12),

$$
\begin{gathered}
\sin \left(\theta_{0}-\Theta_{0}^{-}-\delta\right)-\exp \left(-4 \hat{L} / v_{g}\right) \sin \left(\theta_{0}-\Theta_{0}^{-}+\delta\right)=0, \\
\cos \left(\theta_{0}-\Theta_{0}^{-}-\delta\right)-\exp \left(-4 \hat{L}_{L} / v_{g}\right) \cos \left(\theta_{0}-\Theta_{0}^{-}+\delta\right)=\frac{\Phi_{1} \hat{a}}{R_{0}},
\end{gathered}
$$

with

$$
\Theta_{0}^{-}=R_{0}^{2} \mathrm{e}^{4 \hat{L} / v_{k}} \frac{\left(\alpha_{1}+\beta_{0}\right) \exp \left( \pm 4 \hat{L} / v_{g}\right)+\left(\alpha_{2}+\beta_{0}\right) \exp \left(\mp 4 \hat{L} / v_{g}\right)}{4}
$$

Multiplying (4.26) by itself and replacing (4.27), we eliminate 00 . It follows that

$$
\left(\frac{\Phi_{1} \hat{a}}{R_{0}}\right)^{2}=1-2 \exp \left(-4 \hat{L} / v_{g}\right) \cos \left(\Theta_{0}^{-}-\Theta_{0}^{-}+2 \delta\right)+\exp \left(-8 \hat{L} / v_{g}\right)
$$

where $\Theta_{0}^{+}-\Theta_{0}$ is readily oblaincd invoking (4.28),

$$
\Theta_{0}^{\cdot}-\Theta_{0}=R_{0}^{2} \frac{\alpha_{1}-\alpha_{2}}{4}\left(1-\operatorname{cxp}\left(-8 \hat{L} / v_{g}\right)\right)
$$

Substituting (4.30) into (4.29), we obtain the curves plotted in figure 5 , which yield the size of the steady state, measured by $R_{0}=|A=( \pm \hat{L})|$ (see $(4.20)$ and $(4.24)$ ) in terms of the (rescaled) forcing amplitude. The shape of $A \perp$ is given by (4.24) and depends only on $S$ (through $v_{g}$ ) and $\hat{L}$ (see figure 6). Note that:

(i) These curves are generally multiply S-shaped, showing infinitcly many multiplicity intervals for increasing $\hat{a}$ (in fact, $\hat{a}=a / \sqrt{\varepsilon} \ll 1 / \sqrt{\varepsilon}$ is bounded and only a finite number of these multiplicity intervals apply for a given value of $\varepsilon \ll 1$ ). This is not surprising since multiplicity must be expected in horizontally vibrated containers owing to the interplay between detuning and nonlinearity (see the simpler ODE description by Miles 1984 of horizontal vibrations in a short container). Here, in our large container, as $\hat{a}$ increases ( $R_{0}$ is also increased) the effective detuning $\Theta_{0}^{-}-\Theta_{0}$ varies repeatedly on the interval $[0,2 \pi]$, owing to mismatch between the container length and the wavelength of the effective surface waves (which depends on $R_{0}$, see (4.30)). Each such excursion gives a new multiplicity interval. 

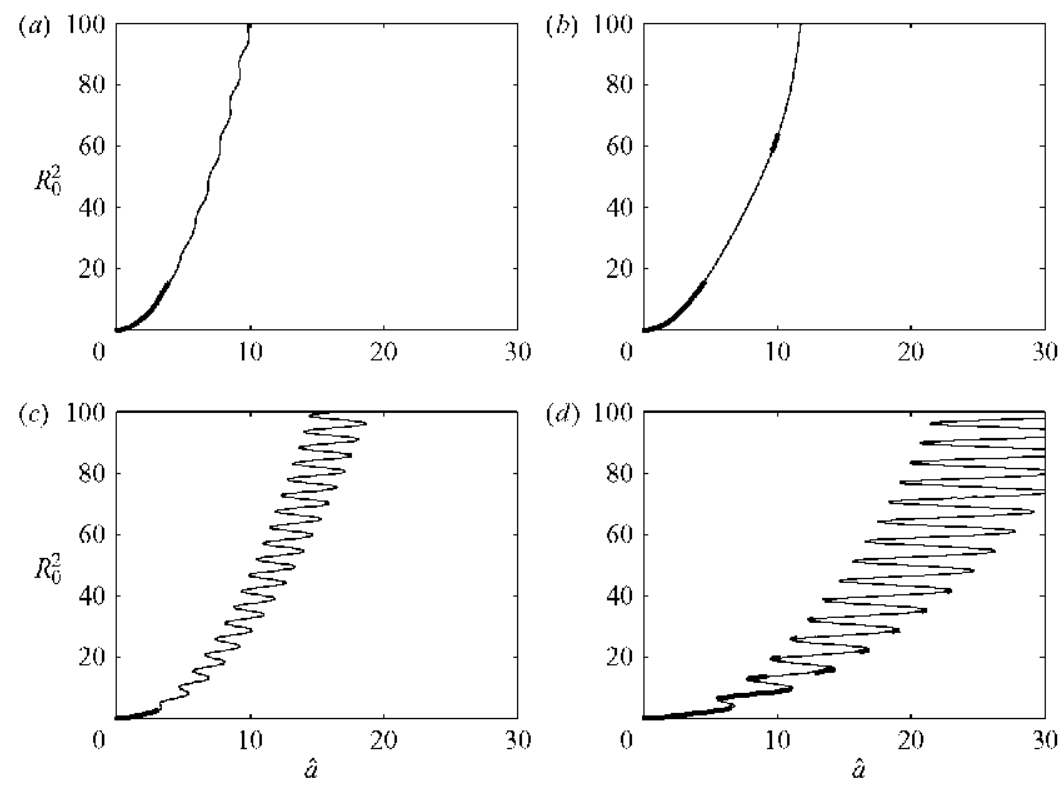

FIGURL: 5. Response curves of the reflection-symmetric steady states of (4.5)-(4.9) and (4.12) (4.14), $R_{0}$ bs. $\hat{a}$ given by (4.29), for $d=3, \hat{L}=0.5, \delta=0$ and (a) $S=0,(b) 0.2$; (c) 0.5 and (d) 0.9 . Stable and unstable stcady states are plotted with thick and thin lines; respectively.

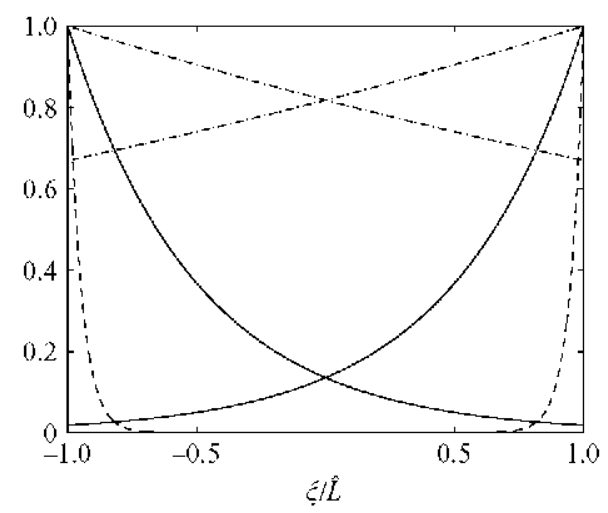

Higlre 6. Amplitudes, $\left|A^{\cdot}(\xi) /\right| A^{+}(\hat{L}) \mid=\exp \left( \pm 2(\xi \pm \hat{L}) / v_{g}\right)$, of the reflection symmetric steady states of $(4.5)-(4.9)$ for $S=0.5\left(v_{g}=1\right)$ and $\hat{L}=0.1(-\cdot-), 1(-)$ and $10(---)$.

(ii) Cubic nonlinearity appears in (4.5) only if

$$
\alpha_{1} \neq \alpha_{2}
$$

as we assume hercinafter; if $\alpha_{1}=\alpha_{2}$, then higher (quintic-)order terms must be considered.

(iii) The results depend quantitatively on the detuning $\delta$, which changes by a $O(1)$ amount under small relative variations of either $L, d$ or $S$ (see (2.1), (2.8) and (4.16) above and recall that $L \gg 1$ ). 
(iv) As a general comment, multiplicity is enhanced by decreasing $\hat{L}=\varepsilon L$. In fact: (a) If $\hat{L}$ is large, the response curve in figure 5 becomes the straight line $R_{0} \simeq \Phi_{1} \hat{a}$, and the solution is unique unless $R_{0} \sim \exp \left(2 \hat{L} / v_{g}\right)$ or larger. Also (see (4.24)), cach countcrpropagating wave vanishes exponentially except in a boundary layer, of $O(1)$-length, near the lateral wall where this wave is created (see figure 6).

(b) If $\hat{L}$ is small, (4.30) yields $\Theta_{0}^{-}-\Theta_{0} \simeq 2\left(\alpha_{1}-\alpha_{2}\right) \hat{L} R_{0}^{2} / v_{g}$. Replacing this into (4.29), we obtain

$$
\frac{\Phi_{1} \hat{a}}{R_{0}} \simeq 2\left|\sin \left[\frac{\left(\alpha_{1}-\alpha_{2}\right) \hat{L} R_{0}^{2}}{v_{g}}+\delta\right]\right|,
$$

if $\left(\alpha_{1}-\alpha_{2}\right) \hat{L} R_{0}^{2} / v_{g}+\delta \neq 0, \pi(\bmod 2 \pi)$, whilc if $\left(\alpha_{1}-\alpha_{2}\right) \hat{L} R_{0}^{2} / v_{g}+\delta \simeq m \pi$ for some integer $m$, then we have

$$
\frac{\Phi_{1} \hat{a}}{R_{0}}=\sqrt{\frac{16 \hat{L}^{2}}{v_{g}^{2}}+4\left[\frac{\left(\alpha_{1}-\alpha_{2}\right) \hat{L} R_{0}^{2}}{v_{g}}+\delta-m \pi\right]^{2}} .
$$

I'hesc expressions show that the response curve cxhibits infinitcly many, fairly wide, multiplicity intervals in this limit. The first multiplicity interval $(m=0)$ is obtained as $\left|\left(\alpha_{2}-\alpha_{1}\right) \hat{L} R_{0}^{2}+\delta\right| / v_{g} \sim \hat{L} \ll 1$, and gives $\hat{a} \sim \hat{L}$. The steady state in this limit is such that the amplitudes of the counterpropagating waves vary only slightly with the horizontal coordinate (see figure 6). Thus $\left|A^{\prime}\right| \simeq|A|$ everywhere and the harmonic wave is almost a standing wave.

(v) The response curves in figure 5 are independent of the parameter $\beta_{0}$, which bears the effect of the mean flow. This parameter does affect stability, which is considered next.

\subsection{Linear stability: harmonic instabilities}

Let $\left(\Lambda^{\perp}, \psi^{m}, f^{m}\right)=\left(\Lambda_{s}^{\circ}, \psi_{s}^{m}, f_{s}^{m}\right)$ be a reflection symmctric steady state of (4.5)-(4.9) and (4.12)-(4.14), given by (4.20)-(4.22), (4.24) and (4.25). We set

$$
\left.\begin{array}{l}
\Lambda^{ \pm}-\Lambda_{s}^{ \pm}=\Lambda_{s}^{ \pm}\left[\left(a^{ \pm}+b=\right) \mathrm{c}^{j \tau}+\left(a^{ \pm}-b^{ \pm}\right) \mathrm{c}^{\bar{\lambda} \tau}\right], \\
\psi^{m}-\psi_{s}^{m}=\psi_{\mathrm{c}^{j \tau}}+\text { c.c., } \quad f^{m}-f_{s}^{m}=F \mathrm{c}^{\lambda \tau}+\text { c.c.. }
\end{array}\right\}
$$

and linearize, to obtain the linear problem (C1) (C7) in Appendix $C$, which is invariant under the following actions, resulting from (4.19),

$$
\begin{array}{ll}
\xi \rightarrow-\xi, & a^{ \pm} \leftrightarrow a^{\mp}, \quad b^{ \pm} \leftrightarrow b^{\mp}, \quad \Psi \rightarrow-\Psi, \\
\xi \rightarrow-\xi, & a^{ \pm} \leftrightarrow-a^{\mp}, \quad b^{ \pm} \leftrightarrow-b^{\mp}, \quad F \rightarrow-F .
\end{array}
$$

Figenmodes invariant under (4.35) and (4.36) will be called reflection symmetric and antisymmetric, respectively. Also, we must distinguish between long- and short-wave instabilitics, which exhibil wavelengths of orders $O(1)$ and $O\left(\varepsilon^{-1 / 2}\right)$, respectively. The difference between both is that dispersion (namely, the term $\mathrm{i} \varepsilon \alpha_{0} A_{\xi \xi}$ in (4.5)) can be neglected for long-wave instabilities, but plays an essential role in shortwave instabilities. Distinction between symmetric and antisymmetric modes is not essential for short-wave instabilities. Both instabilities are numerically analysed in Appendix C.

In order to present the results in a simple way, and to attempt to classify the possible situations, we first note that the linearized stability equations (C 1) (C 7) depend on the steady state through $\left|A_{s}^{-}(\hat{L})\right|=R^{+}(\hat{L})=R_{0}$ (a good measure of the basic steady state since for each $R_{0}$ there is only one steady state as $\hat{a}$ is varied, see figure 5) and 

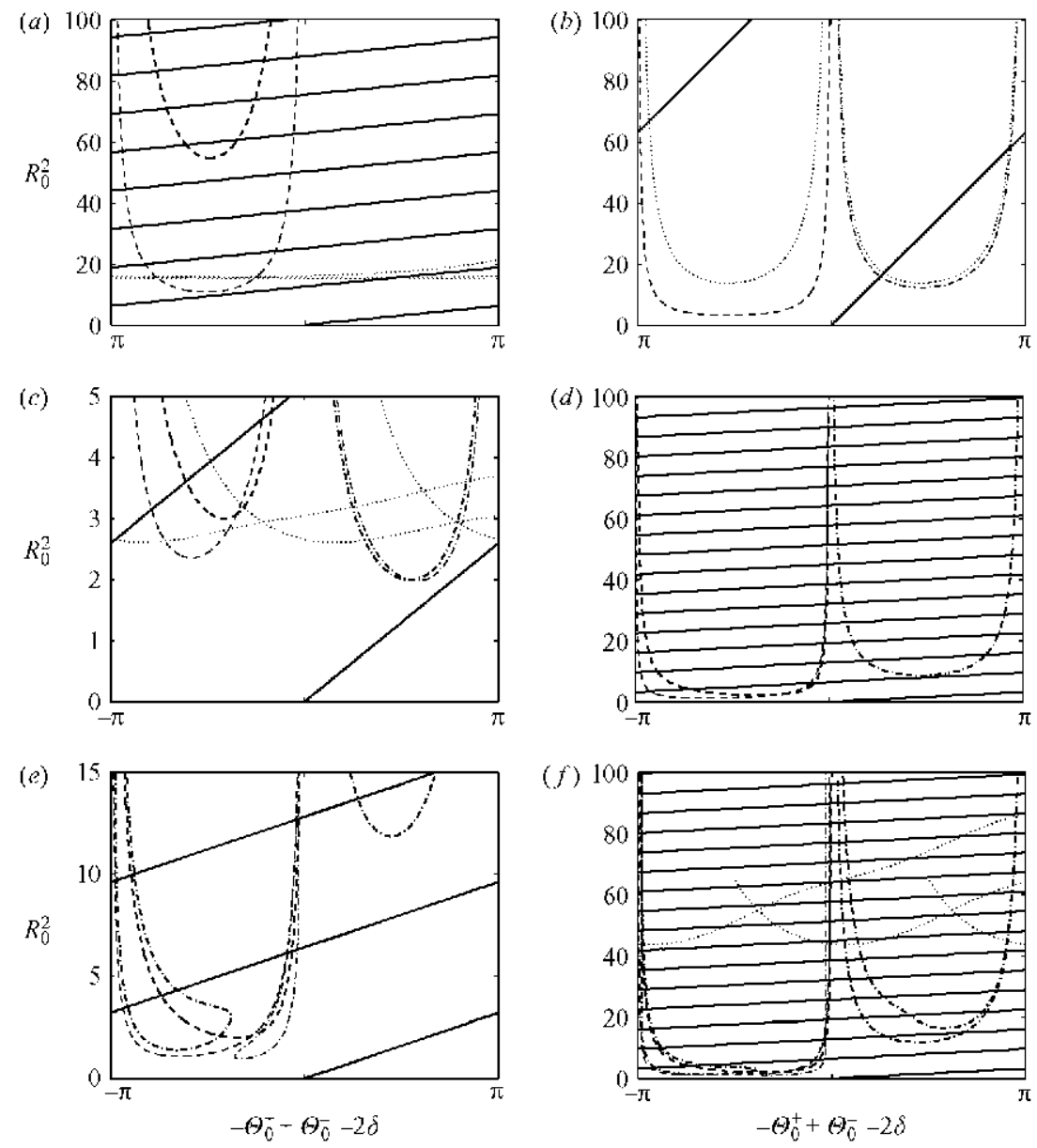

Figidre 7. Marginal instability curves $R_{0} v S . \Theta_{0}^{\prime}-\Theta_{0}^{-}+2 \delta$ for $d=3, \hat{L}=0.5, \delta=0$, and $(a) S=$

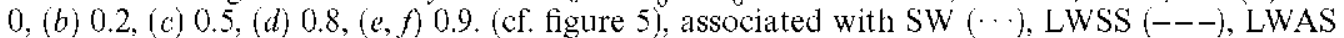
$(---)$, LWSO (-*), and LWAO(-. - l. 'T'he diagram is $2 \pi$-periodic. 'l'he straight thick solid line $(-)$ corresponds to the basic reflection-symmetric steady state obtained for $\delta=0$, and must be translated to the left by an amount $2 \delta$ if $\delta \neq 0$.

$A_{s}^{\prime}(\hat{L}) / A_{s}(\hat{L})=0^{\prime}(\hat{L})-O(\hat{L})=\Theta_{0}^{+}-\Theta_{0}+2 \delta$ (given by $\left.(4.30)\right)$. Thus, for fixed values of $\hat{L}, d$ and $S$, the marginal instability curves (real part of $\lambda=0$ ) can be plotted in the plane $R_{0}$ vs. $\Theta_{0}^{+}-\Theta_{0}^{-}+2 \delta$, and are $2 \pi$-periodic in $\Theta_{0}^{+}-\Theta_{0}^{-}+2 \delta$. Some representative examples are given in figure 7 , where the straight thick line corresponds to the steady statc obtained for $\delta=0$ and must be translated to the Icft by an amount $2 \delta$ as the detuning $\delta$ is varied. According to (4.29)-(4.30), the slope of this straight line depends on $\alpha_{1}-\alpha_{2}$ (plotted in figure 3), and is positive except in the interval $0.21<S<1 / 3$. The remaining lines in figure 7 correspond to the most dangerous instabilities of the various kinds, as indicated in the caption. The long-wave symmetric stationary (LWSS) instability (thick dashed line) corresponds to the turning points of the response curves in figure 5, which yield the end-points of the multiplicity intervals. The remaining instabilities are either short wave (SW), which are always oscillatory, or long wave, which in turn can be either antisymmetric-stationary (LWAS), symmetric-oscillatory (LWSO), or antisymmetric oscillatory (LWAO). LWAS instability yields bifurcation 

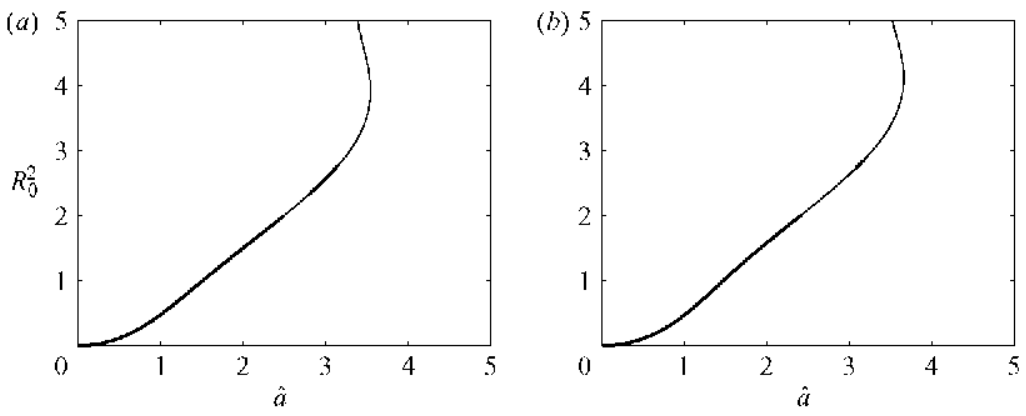

Figitre 8. Responsc curves $R_{0}^{2}$ is. $\hat{a}$ for $\hat{L}=0.5, d=3, S=0.5$ and $(a) \delta=0.25$, (b) 0.4 .

to non-reflection symmetric stcady states, which, invoking (4.1), correspond to nonsymmetric pairs of periodic counterpropagating waves of the system. SW, LWSO and LWAO instabilities instead lead to more complex patterns consisting of pairs of counterpropagating quasi-periodic waves, which can be either reflection symmetric or not. With all these in mind, figure 7 yields the marginal instability points indicated in the response curves in figure 5.

(i) The stability diagram for $S=0.5$ in figure 7 exhibits all the five instabilities mentioned above. The thick straight line intersects first the SW instability line, which is thus the relevant instability, as indicated in figure 5. $\Lambda \mathrm{s} \delta$ is increased, the straight line moves to the left and the nature of the instability changes. For instance, if $0.20<\delta<0.36$, the first instability is LW $\Lambda$ O, whereas it is LWSO in the interval $0.36<\delta<1.11$. In the interval $0.20<\delta<0.475$, there are three instability points, which give two disjoint instability intervals, bounded by two LW and one SW instability points; two examples are plotted in figure 8 that differ only in the nature of the lowest instability point, which is either LWSO or LW $O$, the remaining two being LWAO and SW. At $\delta=0.36$, a competition between LWSO and LWAO instabilities occurs that should give rich dynamics, as are to be expected near $\delta=2.11$, where a competition between two SW instabilitics (with different wavenumbers) occurs. Notc that there are two additional codimension-two points, at $\delta=1.11$ and 2.72 , where SW-LWSO and SW-LW $\Lambda$ S interactions occur.

(ii) For the remaining four values of $S$ in figure 7 , the stability properties of the steady states in figure 5 are obtained in a similar manner. Again, the nature of the instability changes for varying $\delta$. Let us just point out that for $S=0.2$ and 0.8 the response curve exhibits infinitely many disjoint stability intervals, which for $S=0.8$ are interspersed with the multiplicity intervals (cf. figure 5).

From the results described above, we may outline the following conclusions.

1. As $R_{0}$ increases, the first instability can be either short wave or long wave, and in the latter casc, it can be cither steady or oscillatory, and can cither prescrve or break reflection symmetry, depending on the values of the various parameters $(\hat{L}, d$, $S$ and $\delta$ ).

2. The stability properties depend on the detuning $\delta$ (compare the two plots in figure 8), which (as indicated in remark (iii) in $\$ 4.1$ ) is quite sensitive to $L, d$ and $S$.

3. As explained in $\Lambda$ ppendix C.2, the appearance of $\mathrm{SW}$ instabilitics depend on the sign of $\alpha_{0} \hat{\alpha}_{1}$. If $\alpha_{0} \hat{\alpha}_{1}>0$ and is not numerically small (which occurs in the interval $1 / 3<S<3 \sqrt{3} / 2-2$, see figure 18 with $d=3$ ), the SW instability sets in for moderate values of $R_{0}^{2} \sim 1$ and thus competes with the long-wave instabilities. This is the case for $S=0.5$ in figure 7 . If $\alpha_{0} \hat{\alpha}_{1}>0$ is numerically small (which occurs in the intervals 

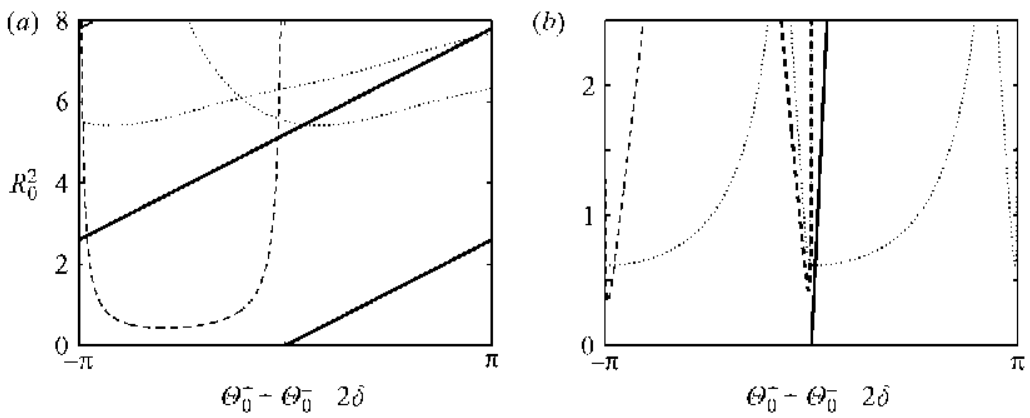

FigLRE 9. As in figure 7 for $S=0.5, \delta=0$. (a) $\hat{L}=0.5, d=10$; (b) $0.01,3$.

$0 \leqslant S<1-\sqrt{3} / 2$ and $0.82<S<1$ if $d=3$, see figure 18), then $S W$ instabilities do appear for large $R_{0}^{2}$. This occurs in figure 7 for $S=0$ and 0.9 . If $\alpha_{0} \hat{\alpha}_{1}<0$ (in the intervals $1-\sqrt{3} / 2<S<1 / 3$ and $3 \sqrt{2} / 2-2<S<0.82$ (see figure 18 with $d=3$ ), then $\mathrm{SW}$ instability curves are either above other long-wave instability curves or are absent, as in the plots for $S=0.2$ and 0.8 in figure 7 .

4. Although we have assumed that $d$ is only logarithmically large, the results above remain valid also for larger values of $d$ provided that $d \ll L$; if $d \sim L$ or larger, then the amplitude equations (4.5) stand, but the viscous mean flow is no longer parallel and $\xi$-derivatives must be added in (4.7) (4.9). If $1 \ll d \ll L$, then LWAS instability appcars as $R_{0}^{2} \sim d^{-1}$ (or invoking (4.29) as $\hat{a} \sim d^{-1 / 2}$ ); this is becausc since $|\psi| \sim d$, that term accounting for coupling to the mean flow becomes of the same order as viscous damping in this limit. This is illustrated in figure 9(a). Short-wave instabilities can appear too (depending on the sign of $\alpha_{0} \hat{\alpha}_{1}$, see figure 18), but require much larger valucs of the forcing amplitude.

5. As $\hat{L} \ll 1$, the marginal instability curves in figure 7 are such that $R_{0}^{2} \sim 1$, which means that $\hat{a} \sim 1$ (see (4.32)), except near some minima, at $\Theta_{0}^{+}-\Theta_{0}+2 \delta \simeq 0$, $\pi(\bmod$ $2 \pi$ ), where $\hat{a} \sim \hat{L}$ (sec (4.33)). Thus, the instability curves (figure 9b) shows quite steep tongues near certain resonance values of the detuning $\delta$, where resonant sloshing modes are excited. In fact, near these resonant values of detuning, the amplitude equations above reduce to a cubic complex ODE, which is similar to that derived by Miles (1984) for horizontally vibrated containers.

\section{Subharmonic instability: subharmonic surface waves}

As in $\$ 3$, the analysis below does not require the assumption that $L \gg d$. The OBГ considered in $\$ 3$ produces an oscillatory normal pressure gradient (see (3.7)) at the frec surface, which is appropriate (through nonlincar interaction with the frec-surface elevation) to parametrically excite subharmonic waves near the free surface. This parametric excitation is standard; the only difference with the Faraday system (Miles \& IIenderson 1990; Tauve 1995) is that now the oscillatory pressure gradient at the free surface is not uniform and thus produces a non-uniform forcing term in the amplitude cquation (namely, the last term in (5.3)). In this section, we obtain the threshold value of the forcing amplitude $a$ for the excitation of these waves, which affect region (b) (figure 1), producing an oscillatory flow such that (cf. (4.1) (4.2))

$$
\begin{aligned}
& f_{S S W}=\mathrm{c}^{\mathrm{i} / / 2}\left[B^{-} \mathrm{c}^{\mathrm{i}(\tilde{k}+\tilde{\delta} / J) x}+B^{-} \mathrm{c}^{-\mathrm{i}(\tilde{k}+\tilde{\delta} / L) x}\right]+\mathrm{c.c} .+\cdots, \\
& \psi_{S S W}=\tilde{\Psi}_{0} \mathrm{c}^{\mathrm{i} / 2 / 2}\left[B^{+} \mathrm{c}^{\mathrm{i}(\tilde{k}+\tilde{\delta} / L) x}-B^{-} \mathrm{c}^{-\mathrm{i}(\tilde{k}-\tilde{\delta} / J) x}\right]+\text { c.c. }+\cdots,
\end{aligned}
$$




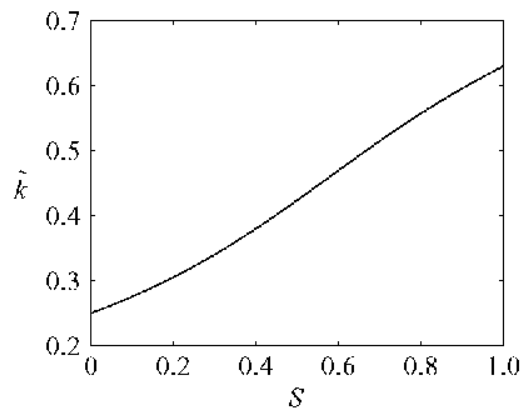

Figiter 10. The wavenumber lor subharmonic waves $\tilde{k}$ in terms of the capillary-gravity balance parameter $S$.

where the spatial detuning $\tilde{\delta}$ is defined below, in (5.6), and the wavenumber $\hat{k}$ and the eigenfunction $\tilde{\Psi}_{0}$ are given by (Vega et al. 2001 and references therein)

$$
(1-S) \tilde{k}+S \tilde{k}^{3}=\frac{1}{4}, \quad \tilde{\Psi}_{0}=\frac{\mathrm{e}^{\tilde{k} y}}{2 \tilde{k}} .
$$

A plot of $\tilde{k}$ vs. $S$ is given in figure 10 . For simplicity, we ignore at the moment both the harmonic surface waves and the mean flow, whose effect is not essential and will be discussed in $\$ 5.3$.

To the linear approximation relevant here, the complex amplitudes $B^{ \pm}$are given by the following amplitude equations and boundary conditions, obtained in Appendices A.2 and B (cf. (4.5), (4.12)),

$$
\begin{gathered}
B_{\bar{T}} \mp B_{\zeta}^{\star}=(-\tilde{d}+\mathrm{i} \tilde{\delta} / \Lambda) B^{-}+\tilde{a} g(\zeta) \bar{B} . \quad \text { in }-\Lambda<\zeta<A, \\
B^{ \pm}=B^{\mp} \quad \text { at } \zeta= \pm \Lambda,
\end{gathered}
$$

in terms of the scaled time variable $T=t /\left(\tilde{v}_{g} d\right)$, the scalcd space coordinate $\zeta$, and the aspect ratio $\Lambda$ defined in (3.2). The scaled damping rate $\tilde{d}$, group velocity $\tilde{v}_{g}$, forcing amplitude $\tilde{a}$, and spatial detuning $\tilde{\delta}$, and the function $g$ are

$$
\begin{gathered}
\tilde{v}_{g}=\omega^{\prime}(\tilde{k}) \equiv 1-S+3 S \tilde{k}^{2}, \quad \tilde{d}=2 \tilde{k}^{2} \varepsilon d / \tilde{v}_{g}, \quad \tilde{a}=a \tilde{k} d / \tilde{v}_{g}, \\
\tilde{\delta}=\tilde{k} L-\alpha\left(2 S \tilde{k}^{3}\right)(\bmod 2 \pi), \quad g(\zeta)=\frac{4}{\pi} \sum_{n=0}^{\infty} \frac{\sinh [(2 n+1) \pi \zeta / 2]}{(2 n+1) \cosh [(2 n+1) \pi \Lambda / 2]}
\end{gathered}
$$

where $\tilde{d}$ has been calculated using (2.12), the quantity $\alpha$ is again as plotted $v s . S$ in figure 3, and the function $g$ is as plotted in figure 11. Equation (5.3) is a balance between inertia, propagation at the group velocity, viscous dissipation, spatial detuning and parametric forcing. As always happens with parametrically excited waves (Tauve 1995), these waves appear upon destabilization of the trivial solution of (5.3)-(5.4), $B^{-}=B^{-}=0$. The instability threshold is obtained seeking non-trivial marginal modes of the form

$$
B==B_{0}^{\perp} \mathrm{c}^{\mathrm{i} \tilde{\omega} I^{\prime}}+B \dot{\bar{l}} \mathrm{c}
$$

where $B_{0}^{ \pm}$and $B_{1}^{ \pm}$are given by

$$
\begin{gathered}
\mathrm{i} \tilde{\omega} B_{0}^{=} \mp B_{0 \zeta}^{\bar{\nu}}=(-\tilde{d}+\mathrm{i} \tilde{\delta} / \Lambda) B_{0}^{=}+\tilde{a} g(\zeta) B_{1}^{\mp} \quad \text { in }-\Lambda<\zeta<A, \\
\mathrm{i} \tilde{\omega} B_{1}^{\mp} \mp B_{1 \zeta}^{\bar{二}}=-(\tilde{d}+\mathrm{i} \tilde{\delta} / \Lambda) B_{1}^{\mp}+\tilde{a} g(\zeta) B_{0}^{\mp} \quad \text { in }-\Lambda<\zeta<\Lambda, \\
B_{0}^{\mp}=B_{0}^{\mp}, \quad B_{1}^{ \pm}=B_{1}^{\mp} \quad \text { at } \zeta= \pm \Lambda .
\end{gathered}
$$




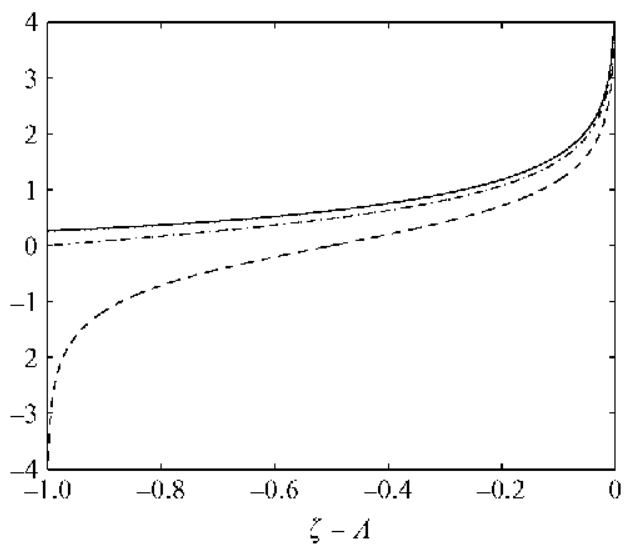

Figurl: 11. The real function $g$ defined in (5.6) for $\Lambda=0.5(---), 1(-,-)$, and $5(\cdots)$, and its asymptotic form $G(-)$ as $\Lambda \rightarrow \infty$, in the lateral region near $\zeta=\Lambda$, given by $(5.11)$. Notc that at $A=5, g$ is almost indistinguishable from its asymptotic valuc.
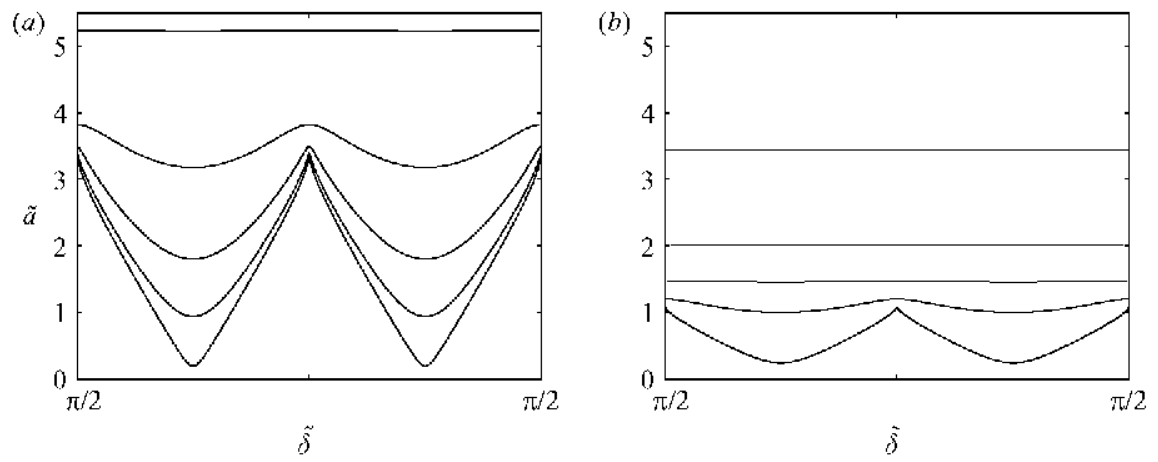

Figurl: 12. Rescaled instability threshold $\tilde{a}=a \tilde{k} d / \tilde{v}_{g}$ vs. $\tilde{\delta}$ for $\tilde{d}=2 \tilde{k}^{2} \varepsilon d / \tilde{v}_{g}=0.1,0.5,1,2,5$ (as $\tilde{d}$ increases, the instability curve moves upwards). The curves are $(\pi / 2)$-periodic in $\tilde{\delta}$. (a) $A=0.5$, (b) 2 .

Since the function $g$ is odd (see (5.6)), this problem is invariant under the actions $\left(\zeta, B_{0}^{ \pm}, B_{1}^{ \pm}\right) \rightarrow\left(-\zeta, B_{0}^{\mp},-B_{1}^{\mp}\right),\left(\zeta, B_{0}^{ \pm}, B_{1}^{ \pm}\right) \rightarrow\left(-\zeta,-B_{0}^{\mp}, B_{1}^{\mp}\right)$, and $\left(\tilde{\omega}, B_{0}^{\mp}, B_{1}^{ \pm}\right) \rightarrow$ $\left(-\tilde{\omega}, \bar{B}_{1}^{ \pm}, \bar{B}_{0}^{ \pm}\right)$. The solutions must be also invariant under these actions, which invoking (5.1) and (5.7) means that the associated patterns are reflection symmetric (namcly, $f_{S S W}(\zeta, t)=f_{S S W}(-\zeta,-t)$ ). 'l'he problem $(5.8)-(5.10)$ is solved numcrically, to obtain the marginal instability curves $\tilde{a}$ ts. $\tilde{\delta}$, plotted in figure 12 and the shapes of $\left|B_{0}^{ \pm}\right|$ and $\left|B_{1}^{1}\right|$ plotted in figure 13 , for various valucs of $\tilde{d}$ and $\Lambda$. Note that the instability curve is reflection symmetric around both $\tilde{\delta}=0$ and $\tilde{\delta}=\pi / 4$, which results from the symmetries $\left(\tilde{\delta}, B_{\overline{0}}^{\perp}, B_{1}^{\perp}\right) \rightarrow\left(-\tilde{\delta}, B_{1}^{\perp}, B_{\overline{0}}^{-}\right)$and $\left(\tilde{\delta}, B_{0}^{\perp}, B_{1}^{\perp}\right) \rightarrow\left(\pi / 2-\tilde{\delta}, \pm B_{1}^{\perp} \mathrm{e}^{ \pm \mathrm{i} \pi \zeta /(2 A)}\right.$, $\left.\mp B_{0}^{ \pm} \mathrm{e}^{\cdot i \pi \zeta(2.1)}\right)$ and also implies that it is $(\pi / 2)$-periodic. In fact, the curve is a sequence of tongues as usual in (Floquet problems appearing in) parametrically excited systems (Fauve 1995), whose minima for varying $\tilde{\delta}$ is attained at $\tilde{\delta}=\pi / 4(\bmod \pi / 2) . \Lambda \mathrm{s}$ expected, either decreasing the damping ratio $\tilde{d}$ or increasing the aspect ratio $A$ has a destabilizing effect. In fact, the analysis above can be simplified in the limit $\Lambda \rightarrow \infty$, but this requires us to consider two distinguished regimes, $\tilde{d} \sim 1$ and $\tilde{d} \sim \Lambda^{1} \ll 1$, which is done in the next two subsections; note that if $\tilde{d} \sim 1$, then 

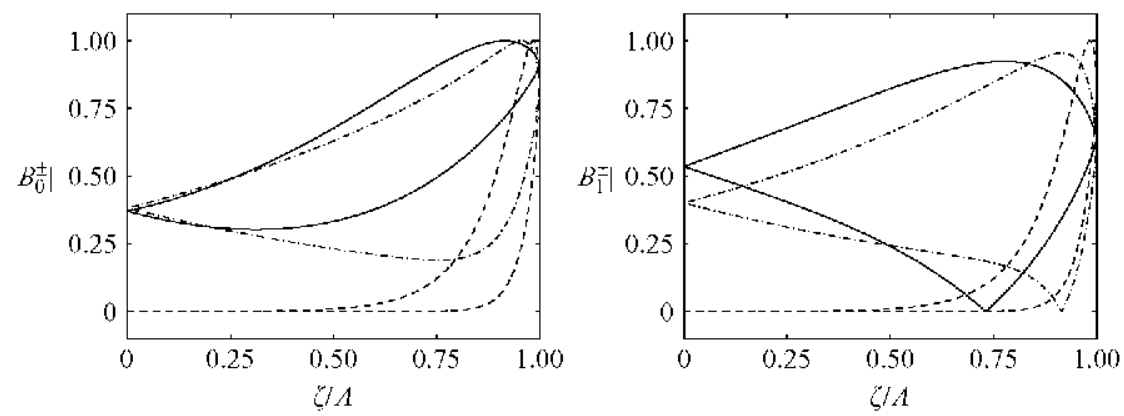

Figurt 13. The amplitudes $B_{0}^{-}(\zeta) \mid$ and $\mid B_{1}^{ \pm}(\zeta)$ vs. $\zeta / \Lambda$ for the harmonic waves given by (5.8) (5.10) for $\tilde{\delta}=0$ and $(\tilde{d}, A)=(1,1)$ i $(1,10)(\quad)$ and $(0.1,10)($. ). Becausc of the symmetries of the problem, (i) only the region $\zeta>0$ need be plotted, (ii) $\left(B_{0}^{1}, B_{1}^{\prime}\right)$ and $\left(B_{0}, B_{1}\right)$ are interchangeable, and (iii) because $\tilde{\delta}=0$ and $\tilde{\theta}=0, B_{0}^{ \pm}$and $B_{1}^{=}$are all real.

activity is concentrated near the latcral walls, whereas the waves penetrate into the bulk if $\Lambda \sim \tilde{d}^{-1} \gg 1$.

$$
\text { 5.1. The limit } \Lambda \rightarrow \infty, \tilde{d} \sim 1
$$

In this limit, the effect of the detuning $\tilde{\delta}$ vanishes at leading order and (5.8) and (5.9) coincide, which implics that $B_{1}^{ \pm} \simeq B_{0}^{ \pm}$. Also, these vanish exponentially except in two $O$ (1)-lateral regions near $\zeta= \pm \Lambda$, which become decoupled and show a similar structure. In these regions, the function $g$ appearing in (5.9)-(5.10) behaves as

$$
g(\zeta)= \pm G(\tilde{\zeta}) \quad \text { with } \tilde{\zeta}= \pm \zeta-\Lambda, \quad G=\frac{4}{\pi} \sum_{n 0}^{\infty} \frac{\exp [(2 n+1) \pi \tilde{\zeta} / 2]}{2 n+1}
$$

(the function $G$ is plotted in figure 11), and $B_{1}^{ \pm} \simeq B_{0}^{ \pm}$are given by

$$
\begin{aligned}
& \mathrm{i} \tilde{\omega} B_{0}^{\perp} \mp B_{0 \tilde{\zeta}}^{\perp}=-\tilde{d} B_{0}^{\perp}+\tilde{a} G(\zeta) B_{0}^{\perp} \quad \text { in }-\infty<\tilde{\zeta}<0, \\
& B_{0}^{\perp}=0 \quad \text { as } \tilde{\zeta} \rightarrow-\infty, \quad B_{0}^{-}=B_{0}^{-} \quad \text { at } \tilde{\zeta}=0 .
\end{aligned}
$$

I'he following asymptotic bchaviour results noting that $G(\tilde{\zeta}) \simeq(4 / \pi) \mathrm{e}^{\pi \tilde{\zeta} / 2}$ as $\tilde{\zeta} \rightarrow-\infty$

$$
B_{0}^{+} \simeq C \mathrm{e}^{(\mathrm{i} \tilde{\omega}+\tilde{d}) \tilde{\zeta}}, \quad B_{0}^{-} \simeq \frac{4 \tilde{a} C \mathrm{e}^{(-\mathrm{i} \tilde{\omega}+\tilde{d}+\pi / 2) \tilde{\zeta}}}{\pi(2 \tilde{d}+\pi / 2)} \text { as } \tilde{\zeta} \rightarrow-\infty,
$$

where $C$ is an arbitrary constant. Using this, we can integrate numerically (5.12)(5.13), to obtain that this problem possesses non-trivial solutions along the marginal instability curve (associated with a stcady instability, $\tilde{\omega}=0$ ), $\tilde{a} \mathrm{vs} . \tilde{d}$, plottcd with a solid line in figure 14. In order to check the approximation in this section, we also plot the exact marginal instability curves, as calculated from (5.8)-(5.10), rescaled in terms of $\tilde{a}=a \tilde{k} d / \tilde{v}_{g}$. Note that the approximation is quite good even for $A=2$, and that $\tilde{a} \rightarrow \pi / 4$ as $\tilde{d} \rightarrow 0$. However, the limil $\tilde{d} \rightarrow 0$ is singular because (sec (5.14)) if $\tilde{d}=0$, then $B_{0}^{+}$does not vanish at the edge of the lateral regions. This leads us to the following limit.

\subsection{The limit $\Lambda \rightarrow \infty, \tilde{d} \Lambda=O(1)$}

Now, the solution at the edge of the lateral regions no longer vanishes, and thus the solution outside these regions, in a region called the bulk region is generally non-zero. 


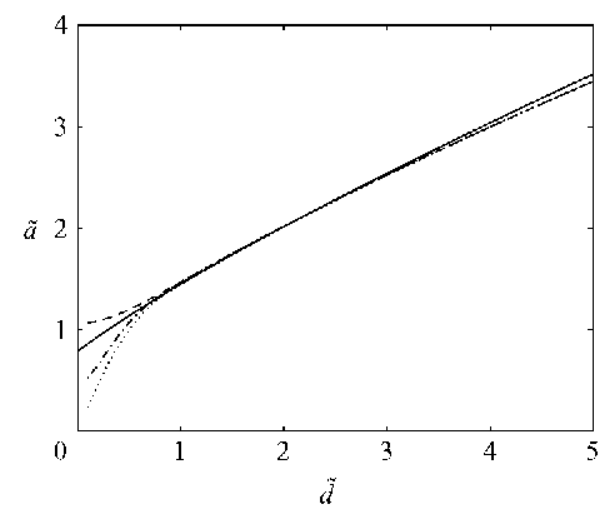

FigirRe 14. Resealed instability threshold amplitude for subharmonic waves $\tilde{a}=a \tilde{k} d / \tilde{v}_{3}$ rs. the rescaled damping ratio $\tilde{d}=2 \tilde{k}^{2} \varepsilon d / \tilde{v}_{g}$ in the limit $A \rightarrow \infty, \tilde{d} \sim 1$. As calculated from (5.12), (5.13) and (5.14) (-); and as calculated from (5.8)-(5.10) (with $\lambda=\mathrm{i} \omega$ ) for $\Lambda=2$ and $\tilde{\delta}=0$ $(---), \pi / 8(--)$ and $\pi / 4(\cdots)$.

\subsubsection{The lateral regions: $\pm \zeta-\Lambda \sim 1$}

In these regions $\tilde{\zeta}= \pm \zeta-\Lambda \sim 1$ and (5.8)-(5.10) lead to (cf. (5.12)-(5.13))

$$
\begin{aligned}
& \mp B_{0 \tilde{\zeta}}^{ \pm}=-\tilde{a} G(\tilde{\zeta}) \bar{B}_{0}^{\mp}, \quad \mp B_{1 \bar{\zeta}}^{\overline{\bar{\zeta}}}=-\tilde{a} G(\tilde{\zeta}) \bar{B}_{1}^{\mp} \quad \text { in }-\infty<\tilde{\zeta}<0, \\
& B_{\overline{0}}^{\perp}=B_{0}, \quad B_{\overline{1}}^{\dot{\overline{1}}}=B_{1}^{\prime} \quad \text { at } \tilde{\zeta}=0, \quad\left|B_{\overline{0}}^{\perp}\right|, \quad\left|B_{\overline{1}}^{\dot{\perp}}\right| \text { bounded as } \tilde{\zeta} \rightarrow-\infty,
\end{aligned}
$$

where we have taken into account that $\tilde{d} \sim \tilde{\omega} \sim 1 / \Lambda \ll 1$. This problem is readily solved in closed form in terms of two arbitrary constants, $C_{0}$ and $C_{1}$, as

$$
\begin{aligned}
& B_{0}^{ \pm}=C_{0} \cos \left(\tilde{a} \int_{\tilde{\xi}}^{0} G(z) \mathrm{d} z\right) \pm C_{1} \sin \left(\tilde{a} \int_{\tilde{\xi}}^{0} G(z) \mathrm{d} z\right), \\
& B_{1}^{ \pm}=C_{1} \cos \left(\tilde{a} \int_{\tilde{\zeta}}^{0} G(z) \mathrm{d} z\right) \pm C_{0} \sin \left(\tilde{a} \int_{\tilde{\xi}}^{0} G(z) \mathrm{d} z\right) .
\end{aligned}
$$

Since $\int_{-\infty}^{0} G(z) \mathrm{d} z=\left(8 / \pi^{2}\right) \sum_{n=0}^{\infty}(2 n+1)^{2}=1$, we have $B_{0}^{\perp}=C_{0} \cos \tilde{a} \pm C_{1} \sin \tilde{a}$ and $B_{1}^{ \pm}=C_{1} \cos \tilde{a} \pm C_{0} \sin \tilde{a}$ as $\tilde{\zeta} \rightarrow-\infty$ or, eliminating the constants $C_{0}$ and $C_{1}$,

$$
B_{0}^{\overline{0}}=B_{0}^{\mp} \pm \tan \tilde{a}\left(B_{1}^{\bar{*}}+B_{1}^{\bar{\mp}}\right), \quad B_{1}^{ \pm}=B_{1}^{\mp} \pm \tan \tilde{a}\left(B_{0}^{ \pm}+B_{0}^{\mp}\right) \text { as } \tilde{\zeta} \rightarrow-\infty,
$$

which gives the boundary condition at $\zeta= \pm \Lambda$ in (5.21) below.

\subsubsection{The bulk region: $\zeta \sim A$}

IIere, the function $g$ vanishes exponentially, and (5.8) and (5.10) become

$$
\mathrm{i} \omega B_{0}^{ \pm} \mp B_{\overline{0 \zeta \zeta}}^{ \pm}=(-\tilde{d}+\mathrm{i} \tilde{\delta} / \Lambda) B_{0}^{ \pm}, \quad \mathrm{i} \omega B_{1}^{ \pm} \mp B_{1 \zeta}^{ \pm}=-(\tilde{d}+\mathrm{i} \tilde{\delta} / \Lambda) B_{1}^{ \pm},
$$

in $-\Lambda<\zeta<\Lambda$, with boundary conditions

$$
B_{0}^{-}=B_{0}^{\perp} \pm \tan \tilde{a}\left(B_{1}^{\perp}+B_{1}^{\prime}\right), \quad B_{1}^{\perp}=B_{1} \pm \tan \tilde{a}\left(B_{0}^{\perp}+B_{0}^{\prime}\right) \quad \text { at } \zeta= \pm \Lambda,
$$

which result from matching conditions with the solutions in the lateral regions, calculated above. Integration of $(5.20)$ yields $\left.B_{0}^{-}=D_{0} \exp \lfloor \pm(\mathrm{i} \omega)+\tilde{d}-\mathrm{i} \tilde{\delta} / A) \zeta\right\rfloor$ and $B_{1}^{ \pm}=$ $D_{1} \exp [ \pm(\mathrm{i} \omega+\tilde{d}+\mathrm{i} \tilde{\delta} / \Lambda) \zeta]$. Substituting these into (5.21), we obtain the following 


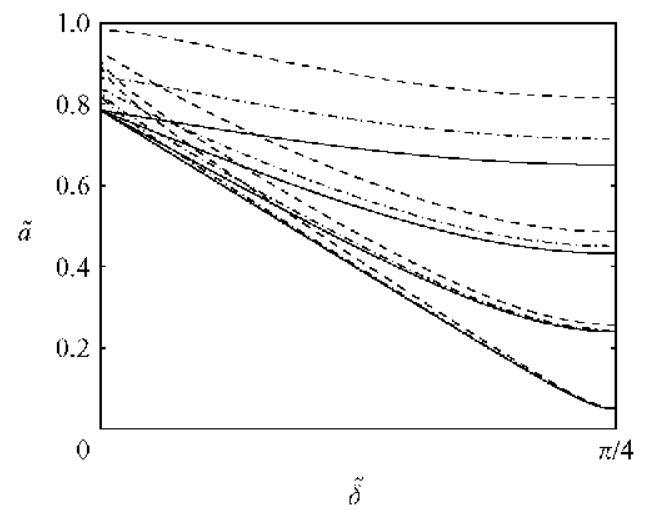

FIGURL 15. Rescaled instability threshold amplitude for subharmonic waves $\tilde{a}=a \tilde{k} d / \tilde{v}_{s} v s$. the spatial detuning $\tilde{\delta}$ for $2 \tilde{d} \Lambda=0.1,0.5,1$ and 2 (the curves move upwards as $2 \tilde{l} A$ increases) in the limit $A \rightarrow \infty, \tilde{d} A \sim 1$. As calculated from (5.24) (-); as calculated from (5.8)-(5.10) for $A=4(---)$ and $\Lambda=10(--)$. Because of the symmetries indicated in connection with figure 12 , we plot only that part of the curve for $0 \leqslant \tilde{\delta} \leqslant \pi / 4$.

homogencous system of lincar equations for the integration constants $D_{0}$ and $D_{1}$

$$
\begin{aligned}
& \sinh (\tilde{d} \Lambda+\mathrm{i} \omega \Lambda-\mathrm{i} \tilde{\delta}) D_{0}-\tan \tilde{a} \sinh (\tilde{d} \Lambda+\mathrm{i} \omega \Lambda+\mathrm{i} \tilde{\delta}) D_{1}=0, \\
& \tan \tilde{a} \cosh (\tilde{d} \Lambda+\mathrm{i} \omega \Lambda-\mathrm{i} \tilde{\delta}) D_{0}-\cosh (\tilde{d} \Lambda+\mathrm{i} \omega \Lambda+\mathrm{i} \tilde{\delta}) D_{1}=0 .
\end{aligned}
$$

This exhibits a non-zero solution provided that the following pair of real equations hold

$$
\begin{gathered}
\left(1-\tan ^{2} \tilde{a}\right) \cos 2 \tilde{\omega} \Lambda \sinh 2 \tilde{d} \Lambda=0, \\
\left(1-\tan ^{2} \tilde{a}\right) \sin 2 \tilde{a} \Lambda \cosh 2 \tilde{d} \Lambda=\left(1+\tan ^{2} \tilde{a}\right) \sin 2 \tilde{\delta},
\end{gathered}
$$

which yields the following instability threshold

$$
\cos 2 \tilde{a}=\frac{\sin 2 \tilde{\delta}}{\cosh 2 \tilde{d} \Lambda} \quad \text { at } \tilde{\omega}=\frac{2 m \pi+1}{2 \Lambda} \quad(m=0, \pm 1, \pm 2, \ldots) .
$$

This provides, for varying $m$, infinitely many tongues (completely similar to those obtained in the Faraday system at quite small viscosity, Miles \& IIenderson 1990), which exhibit the symmetries indicated in the caption of figure 15 , where the instability threshold is plotted with a solid line. In order to check the approximation in this section, we also plot the exact marginal instability curves (calculated from (5.8)(5.10), with $\lambda=\mathrm{i} \omega)$ for $\Lambda=4$ and 10 . Note that the approximation is quite good for $2 \tilde{d} \Lambda=0.1$ (cven for $\Lambda=4$ ) and worsens as $2 \tilde{d} \Lambda$ increases.

For quite small damping, as $\tilde{d} \Lambda \rightarrow 0,(5.24)$ exhibits the asymptotic form $2 \tilde{a} \simeq$ $\pi / 2-2 \tilde{\delta}$ if $|\tilde{\delta}-\pi / 4| \sim 1$ or $\tilde{a} \simeq \sqrt{(\tilde{d} \Lambda)^{2}+(\tilde{\delta}-\pi / 4)^{2}}$ if $|\tilde{\delta}-\pi / 4| \ll 1$. This latter expression gives, in particular, the following asymptotic expression for the minimum of the curve in figure 15 (attained at $\tilde{\delta}=\pi / 4$ )

$$
\tilde{a} \simeq \tilde{d} \Lambda .
$$

5.3. Effects of the harmonic surface waves and the mean flow

IIarmonic surface waves and the mean flow considered in $\$ 4$ have been ignored in the analysis of subharmonic surface waves. They add new terms to the amplitude 


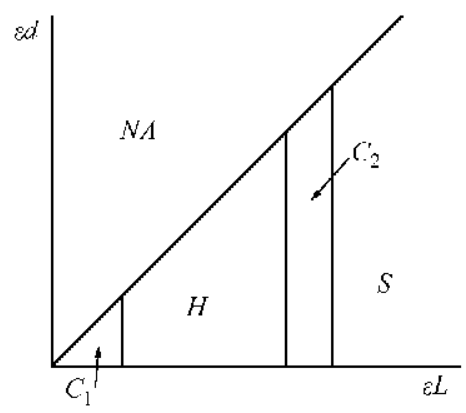

FIGURE 16. Regimes for competition of harmonic and subharmonic instabilities in the plane $\varepsilon L$ vs. $\varepsilon d: C_{1}(\varepsilon d \ll \varepsilon L \ll 1)$, II (either $\varepsilon d \ll \varepsilon L \sim 1$ or $\varepsilon L \gg 1$ and $\left.\sqrt{\varepsilon d} \exp \left(2 \varepsilon L / v_{g}\right) \leqslant 1\right)$ ), $C_{2}\left(\varepsilon L \gg 1\right.$ and $\left.\left.\sqrt{\varepsilon d} \exp \left(2 \varepsilon L / v_{g}\right) \sim 1\right)\right)$, and $S\left(\varepsilon L \gg 1\right.$ and $\left.\left.\sqrt{\varepsilon d} \exp \left(2 \varepsilon L / v_{g}\right) \gg 1\right)\right)$. In region NA (either $\varepsilon d \sim \varepsilon L$ or $\varepsilon d \geqslant \varepsilon L$ ) the analysis of harmonic instabilities above is not applicable.

equations (5.3) which must be rewritten as (cf. (4.5))

$$
\begin{aligned}
B_{T}^{ \pm} \mp B_{\zeta}^{ \pm}= & (-\tilde{d}+\mathrm{i} \tilde{\delta} / \Lambda) B^{\perp}+\tilde{a} g(\zeta) \bar{B}^{\prime} \\
& +\mathrm{i}\left(\alpha_{3}\left|A^{ \pm}\right|^{2}+\alpha_{4}\left|A^{\mp}\right|^{2}\right) B^{-} \pm \mathrm{i} \frac{2 \tilde{k}^{2}}{\tilde{v}_{g}} \int_{-d}^{0} \mathrm{e}^{2 \hat{k} y} \psi_{y}^{m} \mathrm{~d} y B^{ \pm},
\end{aligned}
$$

where $\left(A^{\prime}, A, \psi^{m}\right)$ is a reflection symmetric steady state of (4.5) (4.9), (4.12) (4.14), calculated in $\$ 4.1$, and the coefficients $\alpha_{3} \sim 1$ and $\alpha_{4} \sim 1$ are both real. Now, as happens in Faraday waves (Higuera et al. 2002), the last term is eliminated replacing

$$
B^{ \pm} \rightarrow B^{ \pm} \exp \left[-\mathrm{i} \frac{2 \tilde{k}^{2}}{\tilde{v}_{g}} \int_{0}^{\zeta} \int_{d}^{0} \mathrm{c}^{2 \tilde{k} y} \psi_{y}^{m} \mathrm{~d} y \mathrm{~d} \zeta\right],
$$

which leaves the boundary conditions (5.4) invariant. $A$ similar variable change of the form $B^{ \pm} \rightarrow B^{ \pm} \exp \left\lfloor \pm \mathrm{i} h^{ \pm}(\zeta)\right]$, with $\mathrm{d} h=(\zeta) / \mathrm{d} \zeta=-\left(\alpha_{3}\left|A^{ \pm}\right|^{2}+\alpha_{4}\left|A^{\mp}\right|^{2}\right)$, eliminates the effect of the harmonic surface waves from (5.26), but does affect the boundary conditions. We note that because $A^{-}(\zeta)=A^{-}(-\zeta)$ we have $h^{+}(\zeta)=h^{-}(-\zeta)$, and replacing $B=\rightarrow B=\exp \left[ \pm \mathrm{i} h^{ \pm}(\zeta) \mp \mathrm{i} \hat{\delta} \zeta / \Lambda\right]$, with $\left.\hat{\delta}=-\left[h^{1}(\Lambda)+h(\Lambda)\right] / 2\right)$ leaves the boundary conditions (5.4) invariant, but corrects the spatial decuning as $\tilde{\delta} \rightarrow \tilde{\delta}-\hat{\delta}$. This correction depends on the coefficients $\alpha_{3}$ and $\alpha_{4}$, whose calculation is outside the scope of this paper, and only produces a horizontal shift in the marginal instability curves obtained in $\$ \$ 5$ and 5.2 and plotted in figures 12 and 15 ; those in $\$ 5.1$ instead are independent of detuning.

\section{Competition between harmonic and subharmonic instabilities}

Let us now compare the harmonic and subharmonic instabilities considered above, in $\$ \$ 4.2$ and 5 , to elucidate which one is to appear first for each set of values of the parameters $\varepsilon, d$ and $L$ (satisfying (2.10), which leads to four different regimes, depending only on the parameters $\varepsilon L$ and $\varepsilon d$, as schematically plotted in figure 16 . 'Thesc are illustrated with four possible experiments (figure 17) in an Earth laboratory ( $g=10^{3} \mathrm{~cm} \mathrm{~s}^{-2}$ ) using either a silicone oil such as that used by Kudrolli \& Gollub (1997), with $\nu=0.1 \mathrm{~cm}^{2} \mathrm{~s}^{1}, \sigma=27 \mathrm{dyn} \mathrm{cm}^{1}$ and $\rho=0.85 \mathrm{gcm}{ }^{3}$, or water, with $v=0.01 \mathrm{~cm}^{2} \mathrm{~s}^{-1}, \sigma=72.4 \mathrm{dyn} \mathrm{cm}^{-1}$ and $\rho=1 \mathrm{~g} \mathrm{~cm}^{-3}$. The plots in figure 17 give only the most dangerous instability (namely, the basic reflection-symmetric harmonic pair 


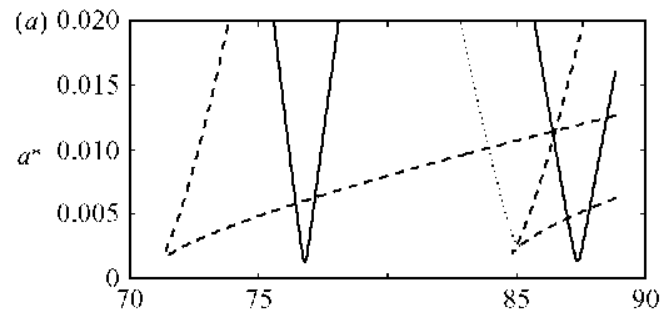

(b)
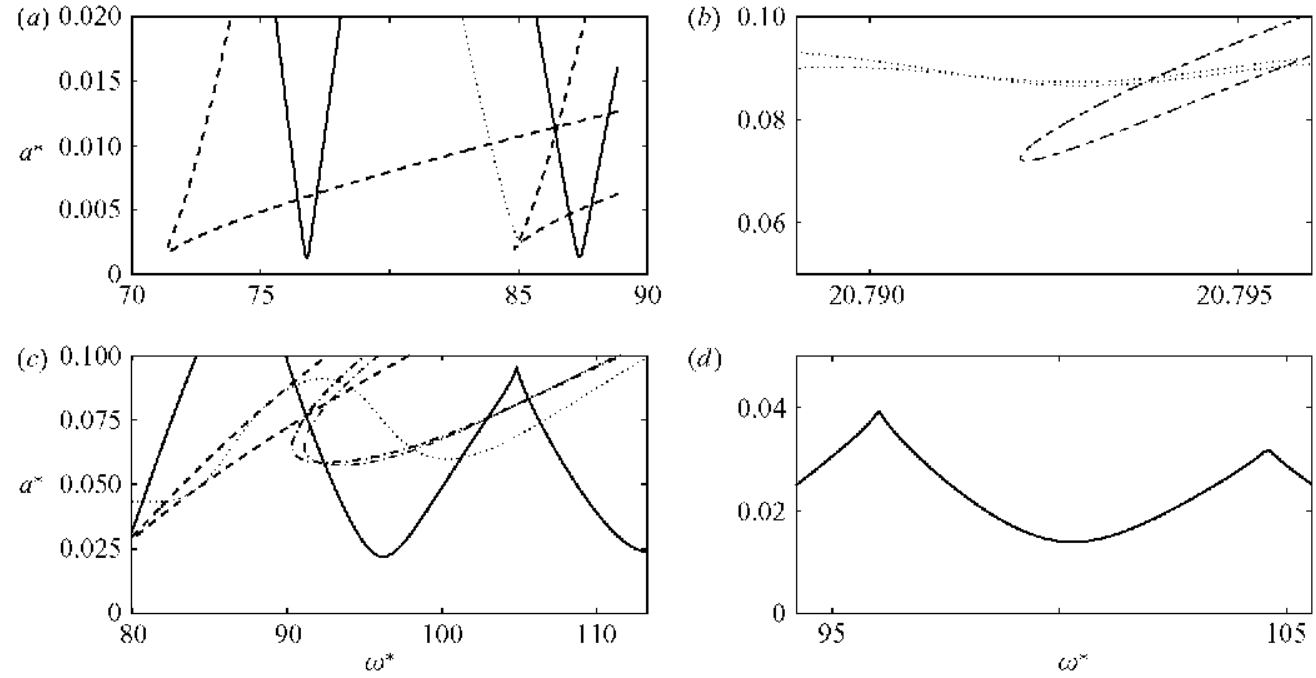

(d)

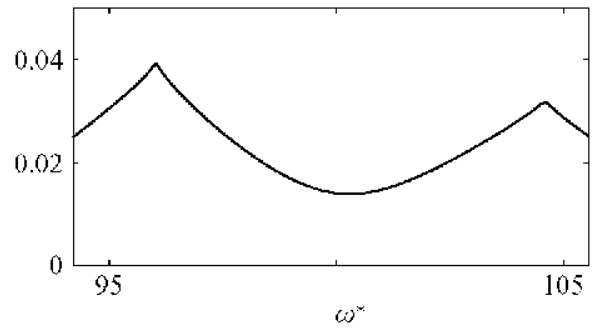

FIGURL 17. Marginal instability curves $a^{*}$ (in $\mathrm{cm}$ ) $v s . \omega^{*}$ (in rad $\mathrm{s}^{-1}$ ) for water in a container with $(a)$ depth $d^{*}=1.78 \mathrm{~cm}$ and horizontal length $2 L^{*}=10.7 \mathrm{~cm}$, and $(b) d^{*}=7 \mathrm{~cm}$ and $2 L^{*}=2.3 \times 10^{4} \mathrm{~cm}$; and siliconc oil with (c) $d^{*}=0.534 \mathrm{~cm}$ and $2 L^{*}=5.34 \mathrm{~cm}$, and (d) $d^{*}=1.78 \mathrm{~cm}$ and $2 L^{*}=10.7 \mathrm{~cm}$. 'Thick solid lines correspond to the subharmonic instability and the remaining lines to the various harmonic instabilities, as indicated in the caption of figure 7. 'lhese experimental conditions are such that all assumptions in the paper are fulfilled. In particular, the slenderness of the system $2 \mathrm{~L}^{*} / \mathrm{d}^{*}$ is always larger than 6 , and the number of surface wave wavelengths, $2 L^{*} /\left(2 \pi \ell^{*}\right)$ is at least 6 . The plotted curves are associated with those instabilitics with the smallest forcing amplitude; when the response curve exhibits multiplicity. associated with the LWSS instability (cusped curve with thick dashed line), the remaining plotted curves correspond to instabilities along the lowest branch on the response curve.

of surface waves is unstable above the curve in figure 17) and are obtained using the non-dimensionalization defined at the beginning of $\$ 2$ and the stability results $\$ \$ 4.2$ and 5 . Because of the effect of harmonic surface waves on the subharmonic instability mentioned in $\$ 5.3$, a horizontal shift of the curve plotted with a solid line is possible that has not been calculated above and must be taken into account when trying quantitative comparison with experiments.

We must distinguish three cases.

(i) If $\varepsilon d \ll \varepsilon L \ll 1$ (region $C_{1}$ in figure 16 ), then both harmonic and subharmonic instabilities do appear for quite small values of $a$, along some resonance tongues whose minima correspond to different forcing frequencies. As explained at the end of $\$ 4$, harmonic instabilities generally occur as $\hat{a} \sim 1$, except near the minima of the LWSS tongues, where $\hat{a} \sim \hat{L} \ll 1$, and as explained in $\$ 5$, subharmonic instabilitics generally require that $\tilde{a} \sim a d \sim 1$, except near the minima, where $\tilde{a} \sim a d \sim \hat{L}=\varepsilon L$. Thus, either harmonic or subharmonic instabilities can appear first, depending on the forcing frequency. An example of a marginal instability curve $\left(a^{*} \text { us. } a\right)^{*}$ ) in this region is ploted in figure $17(a)$, which does give a point in region $C_{1}$ because $(\varepsilon d, \varepsilon L) \sim(0.011,0.034)$; this is obtained (for $\left.\omega^{*} \sim 80 \mathrm{rads}^{-1}\right)$ using $(2.1)$ and $(2.8)$, which gives $\ell^{*} \sim 0.27 \mathrm{~cm}, S \sim 0.5, \varepsilon \sim 0.0017, d=d^{*} / \ell^{*} \sim 6.6, L=L^{*} / \ell^{*} \sim 19.8$. Note that the most dangerous instability is a harmonic long-wave symmetric steady instability (which corresponds to the first instability limit, see $\$ 4.2$ ), except in the interval $81.5<\omega^{*}<84.8$, where it is a harmonic short-wave instability, and in two 
intervals (which are $75.2<\omega^{*}<78.5$ and $86.4<\omega^{*}<88.8$ but can be shifted horizontally, recall our comment above) where it is subharmonic.

(ii) If $\varepsilon d \ll \varepsilon L \sim 1$ (region $\mathrm{H}$ in figure 16) then harmonic instabilities appear as $\hat{a}=\varepsilon^{-1 / 2} a \sim d^{-1 / 2}(\sec (4.33)$ and figures 7 and $9 a)$, and subharmonic instabilitics are triggered as $\tilde{a} \sim a d \sim 1$ (see (5.6) and figures 12, 14 and 15). Thus, harmonic instabilities appear first in this region. The marginal instability curves $a^{*}$ vs. $\omega^{*}$ for a case in this region is ploted in figure 17(b) (now $\omega^{*} \sim 20.8 \mathrm{rad} \mathrm{s}^{-1}$, and using (2.1) and (2.8), $\ell^{*} \sim 2.3 \mathrm{~cm}, S \sim 0.014, \varepsilon \sim 9.1 \times 10^{5}, d=d^{*} / \ell^{*} \sim 3.04, L=L^{*} / \ell^{*} \sim 5 \times 10^{3}$; thus $(\varepsilon d, \varepsilon L)=\left(2.8 \times 10^{-4}, 0.45\right)$ and we are in fact in region $\left.H\right)$. Note that the length of the system is unrealistically large $\left(2 L^{*}=2.3 \times 10^{4} \mathrm{~cm}\right)$ in this experiment, which has been included here only to illustrate that points in region $\mathrm{H}$ do exist. This is because in order to obtain points in region II, an extremely small value of $\varepsilon$ is necessary, which according to (2.8) (2.10) means that the length of the system must be extremcly large (or viscosity extremcly small). For not so small values of $\varepsilon$, regions $C_{1}$ and $C_{2}$ overlap and region II disappears. In order to illustrate this, we consider the response curve in figure 17(c), in which $\omega^{*} \sim 95 \mathrm{rads}{ }^{1}$. Using (2.1) and (2.8), we oblain $\ell^{*} \sim 0.18 \mathrm{~cm}, S \sim 0.5, \varepsilon \sim 0.03$, and $d=d^{*} / \ell^{*} \sim 3, L=L^{*} / \ell^{*} \sim 15$. Thus $(\varepsilon d, \varepsilon L)=(0.09,0.45)$, which formally corresponds to region $H$. In this response curve instcad harmonic and subharmonic instabilitics compete, which corresponds to region $C_{2}$ and formally requires that $\varepsilon L$, be large (see below). Note that the response of the system in this experiment is qualitatively similar to that in figure $17(a)$, the main difference being that the resonance tongues are not so stecp.

(iii) If $\varepsilon L \gg 1$, then harmonic instabilities require that $\hat{a} \sim d^{-1 / 2} \exp \left(2 \varepsilon L / v_{g}\right)$. Subharmonic instabilitics instcad do appear as soon as $\tilde{a} \sim a d \sim 1$, as explaincd in $\$ 5.1$. Thus, we must distinguish three cases.

(i) If $\sqrt{\varepsilon d} \exp \left(2 \varepsilon L / v_{g}\right) \ll 1$, then harmonic instabilities dominate, which corresponds to region II. As above, we have been unable to obtain experimental conditions in this limit, except for unrealistically large lengths of the container.

(ii) If $\sqrt{\varepsilon d} \exp \left(2 \varepsilon L / v_{g}\right) \sim 1$ (region $\left.C_{2}\right)$ then both harmonic and subharmonic instabilities compete. For the sake of brevity, we do not give an experimental point in this region, where response curves are similar to that in figure $17(\mathrm{c})$.

(iii) If $\sqrt{\varepsilon d} \exp \left(2 \varepsilon L_{s} / v_{g}\right) \gg 1$ (region $\mathrm{S}$ ) then subharmonic instabilities do appear first. See figure $17(d)$ for a marginal instability curve in this region, in which since $\omega^{*} \sim 100 \mathrm{rad} \mathrm{s}^{-1}$, we obtain that $\ell^{*} \sim 0.18 \mathrm{~cm}, S \sim 0.5, \varepsilon \sim 0.03, d=d^{*} / \ell^{*} \sim 10$, $L=L^{*} / \ell^{*} \sim 29.7$. Thus $(\varepsilon d, \varepsilon L)=(0.3,0.9)$ and $\varepsilon L$ is not large. In fact, the cxpcrimental conditions in figure $17(d)$ have becn chosen to illustratc that $\varepsilon L$ need not be too large in order to be in this region, provided that $\varepsilon d$ is not too small. This is again because, as indicated above, regions $C_{1}$ and $C_{2}$ overlap (and region II disappears) unless $\varepsilon$ is extremely small.

Summarizing the results:

1. The regimes indicated in figure 16 were obtained from asymptotic considerations that apply as $\varepsilon \rightarrow 0$ and must be reconsidered for small but fixed $\varepsilon$. In fact, in realistic experimental conditions, region $H$ disappears and we have only two regions. Namcly, for fixed $d / L \ll 1$, if $\varepsilon L$ is smaller that a critical valuc, then a compctition between harmonic and subharmonic instabilities occurs, while for larger values of $\varepsilon L$ subharmonic instabilitics dominate for all frequencics.

2. The experimental conditions in figure $17(a, c, d)$ (figure $17 b$ has only an academic value) have been chosen such that the only difference between figures 17(a) and 17(d) is the liquid (same container, similar vibrating frequencics) and the only difference between figure $17(c)$ and $17(d)$ (same liquid, similar vibrating frequencies) is the size 
of the container, and show that increasing either viscosity or the size of the container favours subharmonic instabilities in their competition with harmonic instabilities; of course, increasing viscosity requires larger vibrating amplitudes (compare figures 17(a) and $17(c))$, but increasing the size of the container has only a slight effect on the vibrating amplitude threshold.

3. Increasing the vibrating frequency increases both $\varepsilon d=d^{*} /\left(\omega^{*} \ell^{* 3}\right)$ and $\varepsilon L=$ $L^{*} /\left(\omega^{*} \ell^{* 3}\right)$, which means that the point moves in the diagram in figure 16 towards region $\mathrm{S}$. Thus, increasing 6 favours subharmonic instabilitics. This can be secn in figures $17(c)$ and $17(d)$, but is not further illustrated with a much larger increase of the forcing frequency because the capillary-gravity balance parameter $S$ (sec (2.8)) approaches 1 quite quickly as $\omega^{*}$ is increased, and $S$ must not be too close to 1 for the analysis above of harmonic waves to be valid (surface tension effects have been neglected in the description of the mean flow). The forcing frequency must not, also, be too small, to avoid $\ell^{*}$ being too large, which would violate the assumption that both $L=L^{*} / \ell^{*} \gg 1$ and $d=d^{*} / \ell^{*} \gg 1$.

\section{Concluding remarks}

We have analysed the modulated harmonic and subharmonic surface waves that appear in a large nearly inviscid two-dimensional container subject to horizontal vibration. This has required us to consider an oscillatory bulk flow (OBF, never considered before to our knowledge) and a viscous mean flow (VM 5 , already considered for Faraday waves).

As expected, the system always exhibits a pair of reflection-symmetric counterpropagating harmonic surface waves that are produced by the vibrating lateral walls. The outgoing wave produced at each wall propagates (and decreases by viscous dissipation as it travels) towards the other wall, where it is reflected. IIarmonic waves were analysed in $\$ 4$, where a system of $C \triangle M F$ equations were derived that includes the effects of both the VMT produced by the waves and (implicitly) that of the OBF. The former exhibits its own dynamics, which are coupled to the dynamics of the surface waves; the latter is decoupled and produces that term proportional to $G_{1}$ in (4.16), which is in fact dominant when $d$ is large. The CAM $\Gamma$ equations are not simple, but we claim that they provide the correct weakly nonlinear evolution of harmonic waves, which are steady states of these equations. The primary branch of reflection-symmetric steady states (such as those plotted in figure 6) shows multiplicity (figures 5 and 8) as soon as nonlincar effects come into play. In fact, many (infinitcly many, asymptotically) overlapping multiplicity intervals are possible, but only the first few are relevant because these steady states generally become unstable as the forcing amplitude is increased. Linear stability against harmonic perturbations has been analysed in $\$ 4.2$, where we encountered both long-wave and short-wave instabilities (figures 7 and 9), depending on whether the associated length scale is of the order of the viscous length or the dispersive length. Short-wave instabilities are always oscillatory and reflection symmetric, but long-wave instabilities can be either steady or oscillatory, and either reflection symmetric or antisymmetric.

Subharmonic waves are the relevant ones in vertically vibrated containers, but are not so obviously expected at low amplitude under horizontal vibrations (both subharmonic and superharmonic modes are to be expected in the fully nonlinear regime, for large vibrating amplitude, but this is a different story). We have uncovered the mechanism for the generation of these waves at high frequency, which should also explain the appearance in related systems. Let us remark that subharmonic 
waves have been experimentally observed in vibrated sessile drops (Vukasinovic, Smith \& Glezer 2007) at high vibrating frequency (as compared to the first natural eigen (requency of the system), where they have been secn to be dominant except for quite small vibrating amplitudes, in accordance with our results. The key ingredient in the generation of subharmonic waves is the OBF, which produces an oscillatory normal pressure gradient on the free surface, which is much higher than that produced by the harmonic surface waves. Once the mechanism to excite these waves was clear, we took into account that they are excitcd by a parametric instability, if the forcing amplitude exceeds a threshold value. This has been calculated in $\$ 5$ in the various different regimes that must be considered, depending on the comparative valucs of the container length and depth, and the viscous length (see figures 12,14 and 15). This required only a linear analysis; the associated weakly nonlinear description would be the counterpart of that in $\$ 4$, but is well beyond the scope of the paper. In order to clarify the essential part of the analysis, we ignored the effects of both the harmonic waves and the mean flow in the calculation of the subharmonic instability threshold. As explained in $\$ 5.3$, the former produces only a shift in detuning and the latter has no effect.

The results in $\$ \$ 4$ and 5 have been used in $\$ 6$ to clucidate whether harmonic or subharmonic instabilities appear first, depending on the comparative values of the length and width of the container. The four theoretically possible different regimes for sufficiently small viscosity (figure 16) have been reduced to two in realistic experimental conditions: if both a tremendously high horizontal length of the containcr and an unrcalistically small viscosity (for ordinary liquids, not for example, ${ }^{4} \mathrm{IIe}$ or liquid-vapour $\mathrm{CO}_{2}$, see González-Viñas \& Salam 1994) are to be avoided. We have seen that either harmonic and subharmonic instabilities compete or subharmonic instabilities dominate, the latter being always the case (somewhat surprisingly) as cither the container size or the vibrating frequency are increased.

The results in this paper help to understand the basic issues concerning the initial development of harmonic and subharmonic waves excited by horizontal vibrations in large containers, but leave open several points. In particular, the weakly nonlincar response of subharmonic waves would give more insights into the dynamics of these waves beyond threshold. The various resonances that have becn avoidcd (sec (4.11), (4.18) and (4.31)) assuming generic conditions could give interesting results too. We hope that the analysis in this paper will stimulate experimental work on this system, which is lacking today, in spite of the fact that it constitutes a basic fluid configuration that exhibits quite rich dynamics.

This rescarch was partially supported by the National Acronautics and Space Administration (Grant NNCO4GA47G) and the Spanish Ministry of Fducation (Grants MTM2004-03808 and MTM2004-05796-C02).

\section{Appendix A. Wave reflection and forcing in region (c)}

We consider harmonic and subharmonic waves separately.

\section{A.1. Reflection and forcing of harmonic waves}

In region (c) (see figure 1) we use the variables

$$
\hat{x}=\Lambda-x, \quad \hat{y}=-y, \quad \psi=\hat{\psi} \mathrm{e}^{\mathrm{i} t}+\text { c.c. }, \quad f=\hat{f} \mathrm{e}^{\mathrm{i} t}+\text { c.c. },
$$


to rewrite $(2.2 a),(2.3 a, c)$ and $(2.5 b)$ as

$$
\begin{aligned}
& \hat{\psi}_{\hat{x} \hat{x}}+\hat{\psi}_{\hat{y} \hat{y}} \text { in } 0<\hat{x}<\infty, \quad 0<\hat{y}<\infty, \\
& \mathrm{i} \hat{f}+\hat{\psi}_{\hat{x}}=(1-S) \hat{f}_{\hat{x}}-s \hat{f}_{\hat{x} \hat{x} \hat{x}}-\mathrm{i} \hat{\psi}_{\hat{y}}=0 \quad \text { at } \hat{y}=0, \\
& \hat{\psi}_{\hat{y}}=-a / 2, \quad \hat{f}=0 \quad \text { at } \hat{x}=0, \\
& \hat{f} \text { diverges at most logarithmically as } \hat{x} \rightarrow \infty,
\end{aligned}
$$

where the last condition results from matching conditions with the solution in region (b). As in Nicolás et al. (1998), this problem is solved via sine- and cosine-fourier transforms as

$$
\begin{gathered}
\hat{\psi}=c+C_{1} \hat{x}+C_{2} \sin \hat{x} e^{-\hat{y}}-\frac{a}{2} \hat{y}+\frac{C_{1}+C_{2}}{H_{1}(S)} \int_{0}^{\infty} \frac{e^{\hat{k} \hat{y}} \sin (\hat{k} \hat{x}) \mathrm{d} \hat{k}}{1-(1-S) \hat{k}-S \hat{k}^{3}} \\
-\frac{a}{\pi} \int_{0}^{\infty} \frac{e^{-\hat{k} \hat{y}}[\sin (\hat{k} \hat{x})-\hat{k} \hat{x}] \mathrm{d} \hat{k}}{\hat{k}^{2}\left[1-(1-S) \hat{k}-S \hat{k}^{3}\right\rfloor} \\
\hat{f}=C_{1}+C_{2} \cos \hat{x}+\frac{C_{1}+C_{2}}{H_{1}(S)} \int_{0}^{\infty} \frac{\hat{k} \cos (\hat{k} \hat{x}) \mathrm{d} \hat{k}}{1-(1-S) \hat{k}-S \hat{k}^{3}} \\
-\frac{a}{\pi} \int_{0}^{\infty} \frac{[\cos (\hat{k} \hat{x})-1] \mathrm{d} \hat{k}}{\hat{k}\left[1-(1-S) \hat{k}-S \hat{k}^{3}\right]},
\end{gathered}
$$

where $C_{1}$ and $C_{2}$ arc arbitrary constants and

$$
\begin{aligned}
H_{1}(S) & =-\int_{0}^{\infty} \frac{\hat{k} \mathrm{~d} \hat{k}}{1-(1-S) \hat{k}-S \hat{k}^{3}} \\
& =\frac{(2+S) \cot ^{-1}\left[S / \sqrt{4 S-S^{2}}\right]}{(1+2 S) \sqrt{4 S-S^{2}}}-\frac{\ln S}{2(1+2 S)}>0 .
\end{aligned}
$$

Here, we have taken into account that $0 \leqslant S \leqslant 1$. As $\hat{x} \rightarrow \infty$, (A 7) becomes

$$
\hat{f}=C_{1}+C_{2} \cos \hat{x}+\frac{C_{1}+C_{2}}{H_{1}(S)} \frac{\pi \sin \hat{x}}{1+2 S}+\frac{a}{\pi}\left[\ln \hat{x}-\frac{\pi \sin \hat{x}}{1+2 S}+\gamma+H_{2}(S)\right],
$$

where $y \simeq 0.577$ is the Euler constant and

$$
\begin{aligned}
H_{2}(S) & =\int_{0}^{\infty}\left(\frac{1}{\hat{k}\left[1-(1-S) \hat{k}-S \hat{k}^{3}\right]}-\frac{1}{\hat{k}(1+\hat{k})}\right) \mathrm{d} \hat{k} \\
& =\frac{-S \ln S}{1+2 S}+\frac{2(1-S) \sqrt{S} \cot ^{-1} \sqrt{S /(4-S)}}{(1+2 S) \sqrt{4-S}}>0 .
\end{aligned}
$$

We need only apply matching conditions with the outer solution, given by (2.13) (setting $\int_{\text {ssw }}=\int_{V M H^{\prime}}=0$ ), (3.6), (3.7) and (4.1) to obtain (4.12), with

$$
\begin{aligned}
\alpha(S) & =\tan ^{-1}\left[\frac{4 S}{(1+2 S) H_{1}(S)}\right], \\
G_{1}(S) & =\frac{2 S}{(1+S) \sqrt{16 S^{2}+(1+2 S)^{2} H_{1}(S)^{2}}}, \\
G_{2}(S) & =\frac{(1+2 S) H_{1}(S) \pi+2 S\left(\gamma-H_{2}(S)\right)}{\pi(1+S) \sqrt{16 S^{2}+(1+2 S)^{2} H_{1}(S)^{2}}},
\end{aligned}
$$


where we have taken into account that $H_{1}(S)>0$. These three functions are plotted in figure 4. Water wave reflection at a lateral wall was considered by Hocking (1987).

\section{A.2. Reflection of subharmonic waves}

The only differences with the analysis above are that now $a=0$ and $\mathrm{e}^{\mathrm{it}}$ must be replaced by $\mathrm{e}^{\mathrm{i} t / 2}$ in $(\Lambda 1)$, which requires replacing $\mathrm{i}$ by $\mathrm{i} / 2$ in $(\Lambda 3)$ and $a$ by 0 in (A 4). Alternatively, we can use the new variables and parameters

$$
\tilde{x}=\tilde{k} \hat{x}, \quad \tilde{y}=\tilde{k} \hat{y}, \quad \tilde{\psi}=2 \tilde{k} \hat{\psi}, \quad \tilde{S}=2 \hat{k}^{3} S,
$$

to obtain (A 2)-(A 5) up to notation. Then, the counterpart of (A 9) with $a=0$ yields the boundary condition (5.4), with the detuning $\tilde{\delta}$ as defined in (5.6).

\section{Appendix B. Derivation of the amplitude equation (5.3)}

The derivation of those terms (proportional to $B_{\zeta}^{ \pm}$and $B^{ \pm}$) accounting for transport at the group velocity and viscous damping is standard, as explained in connection with (4.5). Thus, we concentrate in the derivation of the last term on the right-hand side (in particular, of the function $g$ ) that accounts for parametric forcing. To this end, we use the fast and slow timc variables $t$ and $T=t d$, replace $x=d \zeta$, replace (cf. (5.1) and note that the spatial detuning $\tilde{\delta}$ can be set to zero)

$$
\begin{aligned}
& f_{S S W}=\left(B^{\cdot}+a \tilde{F}_{1} B+\cdots\right) \mathrm{c}^{\mathrm{i}(t / 2 \mid \tilde{k} x)}+\left(B+a \tilde{F}_{1} B^{\prime}+\cdots\right) \mathrm{c}^{\mathrm{i}(t / 2 \tilde{k} x)}+\mathrm{c.c.} . \\
& \left.\psi_{S S W}=\left(\tilde{\Psi}_{0} B^{-}+a \tilde{\Psi}_{1} \bar{B}+\cdots\right) \mathrm{c}^{\mathrm{i}(t / 2 \cdot \tilde{k} x)}-\left(\tilde{\Psi}_{0} B+a \tilde{\Psi}_{1} \bar{B}^{\prime}+\cdots\right) \mathrm{c}^{\mathrm{i}(t / 2 \tilde{k} x)}\right]+ \text { c.c. },
\end{aligned}
$$

into (2.2a) (with $\Omega=0),(2.3 a)$ and (2.4), and equate to zero the coefficients of $a \bar{B} \cdot \mathrm{e}^{\mathrm{i}(t / 2 \perp \hat{k} x)}$. The following cquations and boundary conditions result

$$
\begin{aligned}
& \tilde{\Psi}_{1 y y}-\tilde{k}^{2} \Psi_{1}=0 \quad \text { in }-\infty<y<0, \\
& \frac{1}{2} \mathrm{i} \tilde{F}_{1}-\mathrm{i} \tilde{k} \tilde{\Psi}_{1}=-\tilde{k} g(\zeta)-\frac{1}{4} \tilde{k} \varphi_{\zeta}(\zeta, 0), \\
& \mathrm{i} \tilde{k}\left(1-S+S \tilde{k}^{2}\right) \tilde{F}_{1}-\frac{1}{2} \mathrm{i} \tilde{\Psi}_{1 y}=\frac{1}{2} \tilde{k} g+\frac{7}{8} \tilde{k} \varphi_{\zeta}(\zeta, 0), \\
& \tilde{\Psi}_{1}=0 \quad \text { at } y=-\infty .
\end{aligned}
$$

Here, we have taken into account that $\tilde{\Psi}_{0}(0)=1 /(2 \tilde{k})$ (see (5.2)) and the boundary condition (2.4), which to the approximation relevant here can also be written as

$$
(1-S) f_{x}-S f_{x x x}-\psi_{y z}+\psi_{x x t} f+\psi_{x t} f_{x}-\psi_{x} \psi_{x x}+\psi_{y} \psi_{x y}=0,
$$

as obtained by substituting (2.2)-(2.3) into (2.4) and neglecting cubic terms. Note that this boundary condition must be imposed at the boundary, while (B 3) applies at the edge of the viscous boundary layer (region $(d)$ in figure 1), but that the left-hand side of (B 5) is continuous across region (d), and thus can be calculated using the solution in region $(b)$. $\Lambda$ lso, since we are ignoring both viscous damping and the effect of the group velocity, we have set $\partial B=/ \partial \zeta=0$ and $\varepsilon=0$. The boundary condition (B 4) results from matching with the solution in region $(a)$.

Now, the function $g$ is obtaincd imposing that this problem is solvable. Intcgrating (B 1) and imposing (B 4), wc obtain $\tilde{\Psi}_{1}=\mathrm{c}^{\tilde{k} y}$ up to a constant factor. Replacing this and the dispersion relation (5.2a) into (B 3), we obtain

$$
g(\zeta)=\varphi_{\zeta}(\zeta, 0)
$$

and we need only invoke (3.5), to obtain the expression for $g$ quoted in (5.6). 


\section{Appendix C. Linear stability of the reflection symmetric steady states}

l'erturbing around a reflection-symmetric steady state of (4.5) (4.9) and (4.12) (4.14) as indicated in (4.34), and linearizing, we obtain the following lincar problem

$$
\begin{aligned}
& \lambda a^{\perp} \mp v_{g} a_{\xi}^{ \pm}=\mathrm{i} \varepsilon \alpha_{0} b_{\xi \xi}^{ \pm}, \\
& \lambda b^{-} \mp v_{g} b_{\xi}^{\bar{\xi}}=\mathrm{i} \varepsilon \alpha_{0} a_{\xi \xi}^{ \pm}+2 \mathrm{i}\left(\alpha_{1}\left|A_{s}^{\perp}\right|^{2} a^{-}-\alpha_{2}\left|A_{s}^{\prime}\right|^{2} a^{\cdot}\right) \pm 2 \mathrm{i} \int_{-d}^{0} \mathrm{e}^{2 y} \Psi_{y} \mathrm{~d} y, \\
& \lambda \Psi_{y y}=\Psi_{y y y y} \quad \text { in }-d<y<0, \\
& \Psi_{y y}=16\left(\left|A_{s}^{+}\right|^{2} a^{-}-\left|A_{s}^{-}\right|^{2} a^{-}\right), \quad \Psi_{\xi}-\lambda F=4\left(\left|A_{s}^{-}\right|^{2} a^{-}-\left|A_{s}^{+}\right|^{2} a^{-}\right)_{\xi}, \\
& \Psi_{y y y}-\lambda \Psi_{y}+(1-S) F_{\xi}=0 \text { at } y=0, \\
& \Psi=\Psi_{y}=0 \text { at } y=-d \text {, } \\
& A_{s}^{\dot{s}}\left(a^{\dot{ }}+b^{\perp}\right)-A_{s}\left(a^{\cdot}+b^{\prime}\right)=\bar{A}_{s}^{\dot{\circ}}\left(a^{\circ}-b^{\perp}\right)-\bar{A}_{s}\left(a^{\cdot}-b^{\prime}\right)=0 \text {, } \\
& {\left[A_{s}^{\ddagger}\left(a^{ \pm}+b^{\star}\right)\right]_{\xi}+\left[A_{s}^{\mp}\left(a^{\mp}+b^{\mp}\right)\right]_{\xi}=K_{1} a^{+}+K_{2} b^{-}+K_{3} a^{-}+K_{4} b^{-}+K_{5} \int_{-d}^{\infty} \mathrm{e}^{2 y} \Psi_{y} \mathrm{~d} y,} \\
& \left.\left[\bar{A}_{s}^{\dot{s}\left(a^{-}-b^{-}\right)}\right]_{\xi}+\bar{A}_{s}^{\prime}\left(a^{\prime}-b^{\prime}\right)\right]_{\xi}=\bar{K}_{1} a^{+}-\bar{K}_{2} b^{+}+\bar{K}_{3} a^{-}-\bar{K}_{4} b^{-}+\bar{K}_{5} \int_{-i}^{\infty_{i}} \mathrm{e}^{2 y} \Psi_{y} \mathrm{~d} y \\
& \Psi(y=0)=4\left(\left|A_{s}^{-}\right|^{2} a^{-}-\left|A_{s}^{+}\right|^{2} a^{+}\right) \text {at } \xi= \pm \hat{L}, \\
& \int_{-\hat{L}}^{\hat{L}} F(\xi) \mathrm{d} \xi=0
\end{aligned}
$$

where the complex functions $K_{j}=K_{j}(\xi)$ necd not be calculated.

\section{C.1. Long-wave instabilities}

We set $\varepsilon=0$ in (C1) (C2) and ignore the last two boundary conditions in (C6). Steady instabilities $(\lambda=0)$ allow us to solve (C 1$)-(\mathrm{C} 7)$ in closed form, to obtain the following marginal instability curves

$$
\begin{aligned}
& R_{0}^{2}=\frac{4\left[\cos \left(\Theta_{0}^{-}-\Theta_{0}+2 \delta\right)-\cosh 4 \hat{L} / v_{g}\right]}{\left(\alpha_{1}-\alpha_{2}\right)\left[1-\operatorname{cxp}\left(-8 \hat{L} / v_{g}\right)\right] \sin \left(\Theta_{0}^{-}-\Theta_{0}+2 \delta\right)}, \\
& R_{0}^{2}=\frac{4\left[\cos \left(\Theta_{0}^{+}-\Theta_{0}+2 \delta\right)+\cosh 4 \hat{L} / v_{g}\right]}{\left(\alpha_{1}+\alpha_{2}+4 d-8+3 / d^{3}\right)\left[1-\exp \left(-8 \hat{L} / v_{g}\right)\right] \sin \left(\Theta_{0}^{+}-\Theta_{0}+2 \delta\right)},
\end{aligned}
$$

for rellection symmetric and antisymmetric modes, respectivcly.

For oscillatory instabilities $(\lambda \neq 0),(\mathrm{C}, 1)$ can be readily solved to obtain

$$
a^{=}=r_{0}^{\frac{1}{0}} \mathrm{e}^{-\lambda(\xi=\hat{L}) / v_{s}} \text {. }
$$

Substituting this into (C 3)-(C 5), we obtain a linear problem that is solved as

$$
\begin{aligned}
(\Psi, F)= & R_{0}^{2} r_{0}^{\prime}\left(\Psi_{0}, F_{0}\right) \exp \left((4+\lambda)(\xi+\hat{L}) / v_{g}\right) \\
& +R_{0}^{2} r_{0}^{-}\left(-\Psi_{0}, F_{0}\right) \exp \left(-(4+\lambda)(\xi-\hat{L}) / v_{g}\right) \\
& +r_{1}^{\prime}\left(\Psi_{1}, F_{1}\right) \exp (\mu \xi)+r_{1}\left(-\Psi_{1}, F_{1}\right) \exp (-\mu \xi),
\end{aligned}
$$

where $\Psi_{0}, \Psi_{1}$ (which depend on $y$ ), $F_{0}$, and $F_{1}$ (which are constant) are calculated in closed form, but their fairly involved expressions are omitted here. We just point out that those terms proportional to $r_{1}^{-}$represent slowly varying internal waves associated with the mean flow, and exhibit a dispersion relation

$$
(1-S) \mu^{2}[\sqrt{\lambda} d-\tanh (\sqrt{\lambda} d)]=\lambda^{2} \sqrt{\lambda} .
$$


Substituting (C 11) into (C2) (with $\varepsilon=0$ ), and integrating this latter equation, we obtain

$$
\begin{aligned}
b^{\perp}= & r_{2}^{ \pm} \exp \left( \pm \lambda(\xi \pm \hat{L}) / v_{g}\right)+H_{3} r_{0}^{ \pm} \xi \exp \left( \pm(4+\lambda)(\xi+\hat{L}) / v_{g}\right) \\
& +H_{4} r_{0}^{\mp} \operatorname{cxp}\left(\mp(4+\lambda)(\xi+\hat{L}) / v_{g}\right)+H_{5} r_{1}^{ \pm} \operatorname{cxp}( \pm \mu \xi)+H_{6}^{\bar{F}} r_{1}^{=} \operatorname{cxp}(\mp \mu \xi),
\end{aligned}
$$

where the closed-form expressions for the coefficients $H_{3}, \ldots, H_{6}$ are again omitted. Substituting (C 13), (C 10) and (C 11) into the boundary conditions (C6) and (C 7a) gives a homogeneous system of six linear equations in the six unknowns, $r_{0}^{ \pm}, r_{1}^{ \pm}$ and $r_{2}^{\circ}$. Imposing that this system possesses non-trivial solutions provides a complex equation that determines the growth rate $\lambda$. for each set of values of the parameters. Imposing that $\lambda$ be purely imaginary, we obtain the marginal instability curves plotted in figures 7 and 9.

\section{C.2. Short-wave instabilities}

Now we look for slowly modulated waves whose frequency and wavenumber are both large. Thus, the quotient of these two quantities (the phase velocity) must coincide with the group velocity at leading order. It turns out that these waves and the cigenvalue $\lambda$ can be written as

$$
\begin{aligned}
& \left(a^{ \pm}, b^{ \pm}\right)=\left(a_{0}^{\frac{1}{y}}, b_{0}^{\frac{1}{0}}\right) \mathrm{e}^{ \pm \mathrm{i} K_{\xi}^{\xi} / \sqrt{\varepsilon}}+O(\sqrt{\varepsilon}) . \\
& (\Psi, F)=\left(\Psi_{0}^{+}, F_{0}^{-}\right) \mathrm{e}^{\mathrm{i} K \xi / \sqrt{\varepsilon}}+\left(\Psi_{0}^{-}, F_{0}^{-}\right) \mathrm{e}^{-\mathrm{i} K \xi / \varepsilon}
\end{aligned}
$$

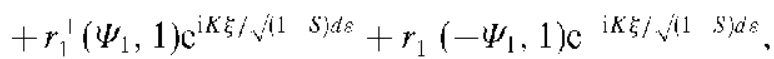

$$
\begin{aligned}
& \lambda=\mathrm{i} v_{g} K / \sqrt{\varepsilon}+\lambda_{1}+O(\sqrt{\varepsilon}),
\end{aligned}
$$

where those terms proportional to $r_{1}^{-}$represent the slowly varying internal waves that also appeared in (C11), and we have taken into account that according to (C12), $\mu \simeq \lambda / \sqrt{(1-S) d}$ as $\lambda \rightarrow \infty$. Note that these internal waves do not affect the surface waves at leading order (namely, no $O(1)$-terms proportional to $r_{1}^{ \pm}$appear on the right-hand side of (C 14)). As a consequence, the internal waves will not play any role in the stability analysis below; they would be needed only to impose the boundary condition (C $6 c)$. Substituting these into (C 1) (C 3), (C 4b, c), (C 5a) and (C $6 a, b)$, we obtain

$$
\Psi_{1}=\sqrt{(1-S) d}(y+d),
$$

and

$$
\begin{aligned}
& \lambda_{1} a_{0}^{ \pm} \mp v_{g} a_{0 \xi}^{ \pm}=-\mathbf{i} \alpha_{0} K^{2} b_{0}^{ \pm}, \\
& \lambda_{1} b_{0}^{ \pm} \mp v_{g} b_{0 \xi \xi}^{ \pm}=-\mathrm{i} \alpha_{0} K^{2} a_{0}^{ \pm}+2 \mathrm{i} \alpha_{1}\left|\Lambda_{s}^{ \pm}\right|^{2} a_{0}^{ \pm} \pm 2 \mathrm{i} \int_{d}^{0} \mathrm{c}^{2 y} \Psi_{0 y}^{ \pm} \mathrm{d} y, \\
& \Psi_{0 y y}^{ \pm}=0 \quad \text { in }-d<y<0,
\end{aligned}
$$

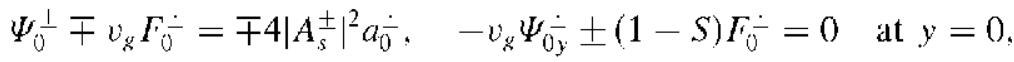

$$
\begin{aligned}
& \Psi_{0}^{ \pm}=0 \text { at } y=-d \text {, } \\
& \left.\begin{array}{l}
\Lambda_{s}^{ \pm}\left(a_{0}^{\perp}+b_{0}^{\perp}\right)=\Lambda_{s}^{\mp}\left(a_{0}^{\prime}+b_{0}\right) \mathrm{c}^{\mp 2 \mathrm{i} K \hat{L} / \sqrt{\varepsilon},}, \\
\bar{A}_{s}^{ \pm}\left(a_{0}^{ \pm}-b_{0}^{ \pm}\right)=\bar{A}_{s}^{\mp}\left(a_{0}^{\mp}-b_{0}^{\bar{y}}\right) \mathrm{e}^{\mp 2 i K \hat{L} / \sqrt{\varepsilon}} \quad \text { at } \xi= \pm \hat{L}_{.} .
\end{array}\right\}
\end{aligned}
$$

IIere, we are ignoring two oscillatory boundary layers, of $O\left(\varepsilon^{1 / 4}\right)$-thicknesses, attached to the free surface and the bottom plate, and two lateral regions attached to the lateral 


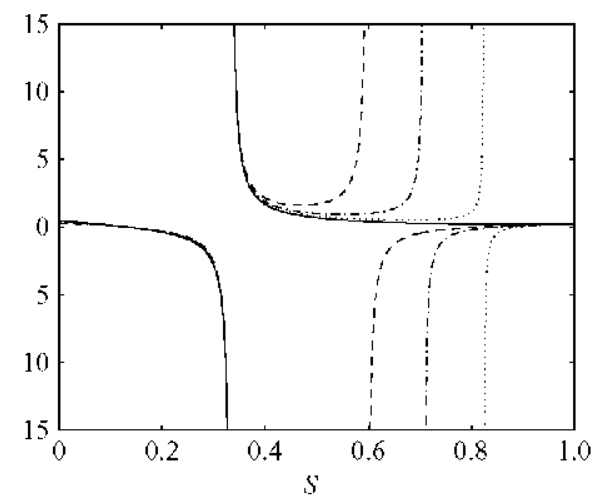

FlGlert 18. The quantity $\alpha_{0} \hat{\alpha}_{1}$ that governs the short-wave instability for $d=3(---)$, $5(),. 10(\cdots)$ and $\infty$ ( )

walls, and only impose inviscid boundary conditions. Also, we are not imposing the boundary condition ( $\mathrm{C} 6 \mathrm{c}$ ), which would be necded only to detcrmine the complex constants $r_{1}^{ \pm}$. The spatial detuning $2 K \hat{L} / \sqrt{\varepsilon}$ can be eliminated upon replacing $a_{0}^{ \pm} \rightarrow$ $a_{0}^{\frac{1}{0}} \mathrm{e}^{\mathrm{i} K \hat{L} \xi / \sqrt{\varepsilon}}$ and $\lambda_{1} \rightarrow \lambda_{1}+\mathrm{i} K \hat{L} / \sqrt{\varepsilon}$, which does not change the stability properties; also, antisymmetric modes turn into symmetric ones replacing $a_{0}^{ \pm} \rightarrow a_{0}^{ \pm} \mathrm{c}^{\mathrm{i} \pi \xi /(2 \hat{\hat{H}})}$ and $\lambda_{1} \rightarrow \lambda_{1}+i \pi /(2 \hat{L})$. Thus, we can replace (C 23) by

$$
A_{s}^{\perp}\left(a_{0}^{ \pm}+b_{0}^{ \pm}\right)=A_{s}^{\perp}\left(a_{0}^{\mp}+b_{0}^{\mp}\right), \quad \bar{A}_{s}^{\perp}\left(a_{0}^{ \pm}-b_{0}^{ \pm}\right)=\bar{A}_{s}^{\perp}\left(a_{0}^{\mp}-b_{0}^{\bar{\mp}}\right) \quad \text { at } \xi= \pm \hat{L},
$$

and consider only symmetric modes. Now, integration of (C.20)-(C.22) yields

$$
F_{0}^{=}=\frac{-4 v_{g}\left|\Lambda_{s}^{-}\right|^{2} a_{0}^{ \pm}}{(1-S) d-v_{g}^{2}}, \quad \Psi_{0}^{ \pm}=\mp \frac{4(1-S)\left|\Lambda_{s}^{\frac{1}{s}}\right|^{2}(y+d) a_{0}^{ \pm}}{(1-S) d-v_{s}^{2}} .
$$

Substituting this into (C 19) and using the cxpression of $\Lambda_{s}^{\perp}$ in (4.20) (with $R_{0}^{\perp}$ given by (4.24)), we obtain

$$
\lambda_{1} b_{0}^{ \pm} \mp v_{g} b_{0 \xi}^{ \pm}=\mathrm{i}\left\{-\alpha_{0} K^{2}+2 \hat{\alpha}_{1} R_{0}^{2} \exp \left\lfloor \pm 4(\xi \mp \hat{L}) / v_{g}\right]\right\} a_{0}^{ \pm},
$$

where

$$
\hat{\alpha}_{1}=\alpha_{1}-\frac{2(1-S)}{(1-S) d-v_{g}^{2}} .
$$

Note that $F_{0}^{ \pm}, \Psi_{0}^{ \pm}$and $\hat{\alpha}_{1}$ diverge as $(1-S) d=v_{g}^{2}$, but this point has been excluded from the analysis, see (4.18). Equation (C27) in conjunction with (C 18) and the boundary conditions (C 24) determines the eigenvaluc $\lambda_{1}$. Since (C 26) has variable coefficients, this problem must be solved numerically, to obtain the results plotted in figures 7 and 9. These numerical results show that if the product $\alpha_{0} \hat{\alpha}_{1}$ (which is plotted in figure 18 for several representative values of $d$ ) is positive, then the instability sets in as $R_{0}$ increases.

\section{C.2.1. The limit $R_{0} \rightarrow \infty$}

Let us rewrite the problem as follows. Since we are only considering symmetric modes, we set

$$
a(\xi) \equiv a_{0}^{+}(\xi) \equiv a_{0}^{-}(-\xi), \quad b(\xi) \equiv b_{0}^{+}(\xi) \equiv b_{0}^{-}(-\xi) \equiv a(\xi) V(\xi),
$$


to rewrite $(\mathrm{C}, 18)$ and $(\mathrm{C}, 26)$ as

$$
\begin{aligned}
& v_{g} a^{\prime}=\left(\hat{\lambda}_{1}+\mathrm{i} \alpha_{0} K^{2} V\right) a, \\
& v_{g} V^{\prime}=-\mathrm{i}\left[\alpha_{0} K^{2}\left(V^{2}-1\right)+2 \hat{\alpha}_{1} R_{0}^{2} \exp \left(4(\xi-\hat{L}) / v_{g}\right)\right] .
\end{aligned}
$$

$\Lambda$ so, the boundary conditions (C 24) yicld, after some algcbra,

$$
\begin{aligned}
& \frac{1+V(\hat{L})}{1-V(\hat{L})}=\exp \left(-2 \mathrm{i}\left(\Theta_{0}^{-}-\Theta_{0}^{+}-2 \delta\right)\right) \frac{1+V(-\hat{L})}{1-V(-\hat{L})}, \\
& \frac{1+V(\hat{L})}{1+V(-\hat{L})}=\exp \left(-\mathrm{i}\left(\Theta_{0}^{-}-\Theta_{0}^{-}-2 \delta\right)-4 \hat{L} / v_{g}\right) \frac{a(-\hat{L})}{a(\hat{L})},
\end{aligned}
$$

where $\Theta_{0}^{-}$and $\Theta_{0}^{\prime}$ are given by (4.28). The sccond condition invoking (C29) leads to the following expression for the eigenvalue $\lambda_{1}$

$$
\lambda_{1}=-2-\frac{v_{g}}{2 \hat{L}}\left[\mathrm{i}\left(\Theta_{0}^{-}-\Theta_{0}^{+}-2 \delta\right)+\frac{\mathrm{i} \alpha_{0} K^{2}}{v_{g}} \int_{-\hat{L}}^{\hat{L}} V(\xi) \mathrm{d} \xi+\ln \frac{1+V(\hat{L})}{1+V(-\hat{L})}\right],
$$

where $V$ is calculated numerically from (C 30)-(C 31). Now, we take the limit

$$
|V| \sim R_{0} \gg 1, \quad K \sim 1
$$

in (C 30), in which

$$
V \simeq-\frac{\sqrt{-2 \alpha_{0} \hat{\alpha}_{1}} R_{0} \operatorname{cxp}\left(2(\hat{L}-\xi) / v_{g}\right)}{\alpha_{0} K}
$$

This expression applics except in two boundary laycrs near $\xi= \pm \hat{L}$, which are required to satisfy the boundary condition (C 31). Substituting this into (C.33), we obtain

$$
\lambda_{1}=\frac{v_{g} K R_{0} \sqrt{\alpha_{0} \hat{\alpha}_{1}}\left(1-\exp \left(-4 \hat{L} / v_{g}\right)\right)}{4 \hat{L}},
$$

which shows that these small scales are destabilizing for sufficiently large $R_{0}$ if $\alpha_{0} \hat{\alpha}_{1}>0$. 'Thus, if $\alpha_{0} \hat{\alpha}_{1}>0$, then the stcady statc is unstable for sufficicntly large $R_{0}$, for arbitrary values of the remaining parameters. If instead $\alpha_{0} \hat{\alpha}_{1}$ exhibits the opposite sign, then the right-hand side of (C 36) is purcly imaginary and we do not come to any conclusion from the analysis above; the calculation of the real part of $\lambda_{1}$ would require us to consider higher-order terms. Note that the instability is enhanced by increasing cither $\alpha_{0} \hat{\alpha}_{1}$ or $R_{0}$.

\section{REFERENCES}

ARANSON, I. S. \& TSIMRING, L. S. 2006 Patterns and collective behavior in granular media: theoretical concepts. Ren. Mod. Phys. 78, 641692.

Batchelor, G. K. 1967 An Introduction to Fhid Mechanics. Cambridge University Press.

BechHoffer, J., Ego, V., Manneville, S. \& Johnson, B. 1995 An experimental study of the onset of parametrically pumped surface waves in viscous fluids. J. Fituid Mech. 288, 325-350.

Billngham, J. 2002 Nonlincar sloshing in zero gravily. J. Fluid Mech. 464, 365-391.

Cross, M. C. \& Hohenberg, P. C. 1993 Pattern lormation outside of cquilibrium. Rev. Mod. Phys. $65,851-1112$.

DANIIIS, P. G. 1978 Finite amplitude two-dimensional convection in a finite rotating system. Proc. R. Soc. Lond. A 363, 195-215.

DAvi:y, A. \& Sillwakisos, S. 1974 On three-dimensional packets of surface waves. Proc. R. Soc. Lond. A 338, 101-110. 
Faltinsen, O. M. \& Timokha, A. N. 2002 Asymplotic modal approximation of nonlinear resonant sloshing in a rectangular tank with small fluid depth. J. 1'tuid Mech. 470, 319-357.

Faltinsen, O. M., Rogrebakke, O. F. \& Timokha, A. N. 2006 Transicnt and slcady-statc amplitudes of resonant threc-dimensional sloshing in a squarc-based tank with a linite lluid depth. Phys. likids 18, 012103 .

Faraday, M. 1831 On the forms and states assumed by lluids in contact with vibrating clastic surfaces. Phil. Trans. R. Soc. Lond. 121, 319-340.

FaUve, S. 1995 Paramotric instabilitics. In Dynamics of Nonlinear and Disordered Systems (cc. G. Martincz-Mckler \& T. H. Scligman), pp. 67-115. World Scientilic.

FENG, Z. C. 1997 Transition to travelling waves from standing waves in a rectangular container subjected to horizontal cxcitations. Phys. Rev. Lett. 79, 415-418.

Funakoshi, M. \& Inote, S. 1988 Surlace waves due to resonant horizontal oscillation. J. Flaid Mech. 192, 219-247.

Gavruyuk, I., Lomsovsкy, I. \& Tімокin, A. 2004 ' 'wo-dimensional variational vibroequilibria and Faraday's drops. Z. Antgew. Math. Phys. 55, 1015-1033.

GoN/ÁI:\%-VIÑAs, W. \& SAI.AM, .T. 1994 Surface waves periodically excited in a $\mathrm{CO}_{2}$ tube. Europhys. Lett. 26, 665-670.

HI:NIIIRSON, D. M. \& MII.IS, J. W. 1994 Surface wave damping in a circular cylinder with a fixed contact line. J. Fluid Mech. 213, 285299.

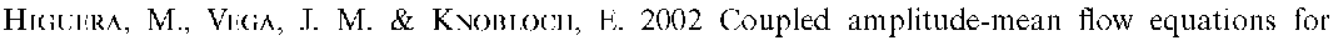
nearly-inviscid Faraday waves in moderate aspect ratio containers. J. Nonlin. Sci. 12, 505551.

IIILL, D. F. 2003 Transient and steady state amplitudes of forced waves in rectangular basins. Phys. Fluids 15, 15761587.

Ilocking, L. M. 1987 Reflection of capillary-gravity waves. Wave Molion 9, 217-226.

Ivanova, A., Kozlov, V. \& Evesoue, P. 1996 Patlerning of liquilicd sand surlace in a cylinder lilled with liquid and subject to horizontal vibrations. Europhys. Lelt. 35, 159-164.

JAEGer, H. M. \& Nagel, S. R. 1996 Granular solids, liquids, and gases. Rev. Mod. Phys. 68, $1250-1273$.

KozLov, V. G. 1991 Experimental investigation of vibrational convection in pseudoliquid layer. In IIydrodynamics and Ileat Transfer in Micrograbily (ed. G. Avduevsky), pp. 57-61. Gordon and Breach.

Kudrolli, A. \& Gollub, J. P. 1997 Patterns and spatio-temporal chaos in parametrically forced surface waves: a systematic survey at large aspect ratio. Physica I) 97, 133154.

LAPUERTA, V., MANCEBO, F. J. \& VEGA, J. M. 2001 Control of Rayleigh-Taylor instability by vertical vibration in large aspect ratio containers. Phys. Rev. H. 64, 016318-1-17.

Lapuerta, V., Martel, C. \& VEGA, J. M. 2002 Interaction of nearly-inviscid liaraday waves and mean flows in 2-D containers of quite large aspect ratio. Physica D 173, 178-203.

MARTEL, C. \& VEGA, J. M. 1996 l'inite size effects near the onset of the oscillatory instability. Nonlinearity 9, 1129-1171.

Martel, C. \& VEGA, J. M. 1998 Dynamics of a hyperbolic system that applics at the onset of the oscillatory instability. Nonlinearily 11, 105-142.

Martel, C., Vega, J. M. \& KNoBloch, K. 2003 Dynamics ol countcrpropagating waves in parametrically driven systems: dispersion vs. advection. Physica D 174, 198-217.

Miles, J. W. 1984 Resonantly lorecd surlace waves in a circular cylinder. J. Fluid Mech. 149, 15-31.

Mitis, .T. \& Hinidirson, D. 1990 l’arametrically forced surface waves. Amu. Rev. Fluid Mech. 22, $143-165$.

Nı:wit.., A. C. 1985 Solitons in Mathematics and Physics. Society for Industrial and $\Lambda$ pplied Mathematics.

Nicor.́s, J. A., Rivas, D. \& Vicis, J. M. 1998 On the steady streaming flow due to high frequency vibration in nearly-inviscid liquid bridges. J. Fluid Mech. 354, 147174.

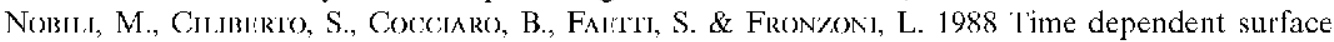
waves in a horizontally oscillating container. Huro. Phys. Lett. 7, 587592.

OCKENDON; J. R. \& OCKENDON, II. 1973 Resonant surface waves. J. Hinid Mech. 59, 397-413.

RAYLEIGH, LORD 1883 On the crispations of fluid resting upon a vibrating support. Phil. Mag. 16, $50-58$.

Riley, M. 2001 Stcady strcaming. Anmu. Rev. Fluid Mech. 33, 43-65.

Ristow, G. H. 2000 Pattern Formation in Gramular Materials. Springer. 
Schlichting, H. 1968 Boundary Layer Theory. McGraw-Hill.

VeGA, J. M., KNobloch, E. \& Martel, C. 2001 Nearly inviscid Faradiay waves in annular containcrs ol moderately large aspect ratio. Physica D 154, 147-171.

Vukasinovic; B., Smiti, M. K. \& Gilizer, A. 2007 I ynamics of a sessile drop in forced vibration. J. Fluid Mech. (in press).

Worle, G. H. 1969 The dynamic stabilization of the Rayleigh laylor instability and the corresponding dynamic cquilibrium. Z. Phys. 227, 291-300.

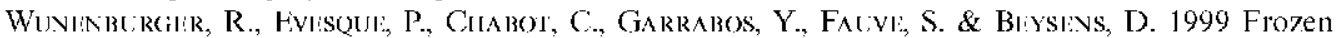
wave induced by high frequency horizontal vibrations on a $\mathrm{CO}_{2}$ liquid gas interface near the critical point. Phys. Rev. E 59, 5440-5445. 